\title{
A Framework for Brain Atlases: Lessons from Seizure Dynamics
}

\author{
Andrew Y. Revell ${ }^{*}, 1,2, a$, Alexander B. Silva $2,3,4, a$, T. Campbell Arnold ${ }^{2,3}$, Joel M. Stein ${ }^{2,6}$, Sandhitsu R. Das ${ }^{2,5}$, \\ Russell T. Shinohara ${ }^{7,8}$, Dani S. Bassett ${ }^{1,2,3,5,10,11,12,13}$, Brian Litt ${ }^{2,3,5}$, and Kathryn A. Davis ${ }^{1,2,5}$ \\ ${ }^{1}$ Department of Neuroscience, Perelman School of Medicine, University of Pennsylvania, Philadelphia, PA 19104 USA \\ ${ }^{2}$ Center for Neuroengineering and Therapeutics, University of Pennsylvania, Philadelphia, PA 19104 USA \\ ${ }^{3}$ Department of Bioengineering, School of Engineering and Applied Science, University of Pennsylvania, Philadelphia, PA 19104 USA \\ ${ }^{4}$ Medical Scientist Training Program, University of California, San Francisco, CA 94143 USA \\ ${ }^{5}$ Department of Neurology, Perelman School of Medicine, University of Pennsylvania, Philadelphia, PA 19104 USA \\ ${ }^{6}$ Department of Radiology, Perelman School of Medicine, University of Pennsylvania, Philadelphia, PA 19104 USA \\ ${ }^{7}$ Department of Biostatistics, Epidemiology, and Informatics, Perelman School of Medicine, University of Pennsylvania, Philadelphia, PA 19104 USA \\ ${ }^{8}$ Penn Statistics in Imaging and Visualization Endeavor, Perelman school of Medicine, University of Pennsylvania, PA 19104 USA \\ ${ }^{9}$ Center for Biomedical Image Computing and Analytics, Perelman School of Medicine, University of Pennsylvania, PA 19104 USA \\ ${ }^{10}$ Department of Electrical and Systems Engineering, School of Engineering and Applied Science, University of Pennsylvania, Philadelphia, PA 19104 USA \\ ${ }^{11}$ Department of Physics and Astronomy, College of Arts and Sciences, University of Pennsylvania, Philadelphia, PA 19104 USA \\ ${ }^{12}$ Department of Psychiatry, Perelman School of Medicine, University of Pennsylvania, Philadelphia, PA 19104 USA \\ ${ }^{13}$ Santa Fe Institute, Santa Fe, NM 87501 \\ a These authors contributed equally \\ * Corresponding author: andrew.revell@pennmedicine.upenn.edu
}

\begin{abstract}
Brain maps, or atlases, are essential tools for studying brain function and organization. The abundance of available atlases used across the neuroscience literature, however, creates an implicit challenge that may alter the hypotheses and predictions we make about neurological function and pathophysiology. Here, we demonstrate how parcellation scale, shape, anatomical coverage, and other atlas features may impact our prediction of the brain's function from its underlying structure. We show how network topology, structure-function correlation (SFC), and the power to test specific hypotheses about epilepsy pathophysiology may change as a result of atlas choice and atlas features. Through the lens of our disease system, we propose a general framework for atlas selection. This framework aims to maximize the descriptive, explanatory, and predictive validity of an atlas. Broadly, our framework strives to provide empirical guidance to neuroscience research utilizing the various atlases published over the last century.
\end{abstract}

Brain Atlas | Networks | Epilepsy | Structure-function

\section{Introduction}

How we define anatomical brain structures and relate those structures to the brain's function can either constrain or enhance our understanding of behavior and neurological diseases ${ }^{1-4}$. Discoveries by scientists like Carl Wernicke and Pierre Paul Broca, who mapped specific brain regions to speech function, in addition to case studies from Phineas Gage and H.M., who lost specific brain regions with resultant changes in brain function and behavior, exemplify how brain structure and function are fundamentally linked ${ }^{5-7}$. Properly labeling brain structures is paramount for enabling scientists to effectively communicate about the variability between healthy individuals and about the regions involved in neurological disorders $^{8}$. Yet, no consensus has been reached on the most appropriate ways to label and delineate these regions, as evident by the wide variety of brain maps, or atlases, defining neuroanatomical structures ${ }^{9}$.

In common usage, an atlas refers to a "collection of maps" 10 that typically defines geo-political boundaries and may include coarse borders (continental), fine borders (city), and anything in between (country; Fig. 1a, left). Borders ${ }^{11}$ are usually consistent across atlases of the world. In contrast, atlases of the brain are not consistent. Four separate atlases (Fig. 1a, right) may define the superior temporal gyrus differently. For example, over ninety percent of the anterior superior temporal gyrus in the Harvard-Oxford atlas ${ }^{16}$ overlaps with the posterior superior temporal gyrus in the Hammersmith atlas ${ }^{17}$. Atlases may also differ in other ways, including the parcellation size, neuroanatomical coverage, and complexity of brain region shapes. For instance, the Yeo atlas ${ }^{18}$ contains 7 or 17 parcels while the Schaefer atlases ${ }^{19}$ may have between 100 and 1,000 parcels. Complicating matters further, atlases can differ in their intended use. The MMP atlas ${ }^{20}$ was intended for surfacebased analyses ${ }^{21}$, yet a volumetric version (without subcortical structures) was independently created and used in connectivity studies $^{22}$. The plethora of available atlases poses a problem for reproducibility in studying healthy and diseased populations and for metanalyses describing the involvement of different regions of the brain in various diseases. This has been termed the Atlas Concordance Problem ${ }^{4}$.

In the present study, we perform an extensive evaluation of the available atlases in the neuroscience literature (Table 1 ) by examining the effect of varying features such as parcellation size, coverage, and shape (Fig. 1b) on structural connectivity (Fig. 1c) and structure-function correlation (SFC; Fig. 1d). Note the important distinction between the terms atlas, template, and stereotactic space ${ }^{9}$ (see Fig. S1). We utilize a total of 52 brain atlases, including many routinely used in common neuroimaging software, and found that different atlases may alter the power to test a hypothesis about epilepsy pathophysiology that seizures propagate through the underlying structural connections of the brain. This hypothesis has been previously supported in prior research ${ }^{13,14,23,24}$

In the context of our experimental design, we propose a new framework outlining how to appropriately choose an atlas 
a

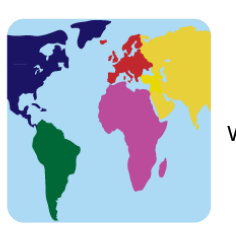

Atlas

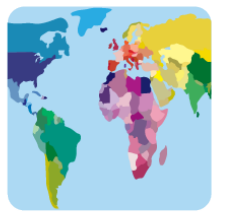

Superior Tempora

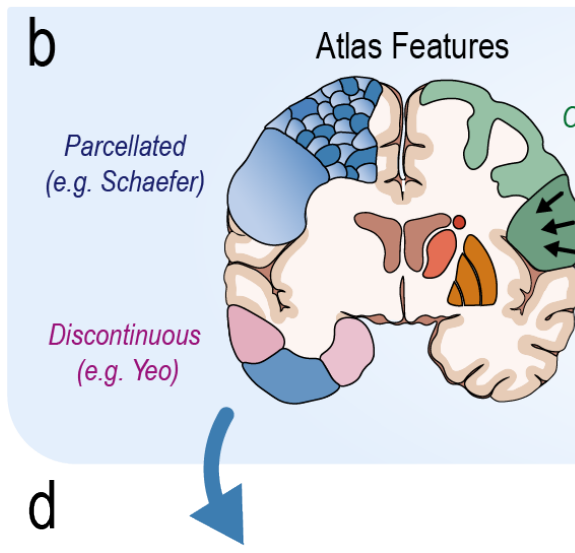

i

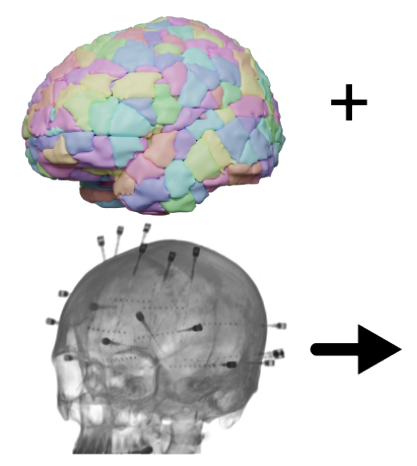

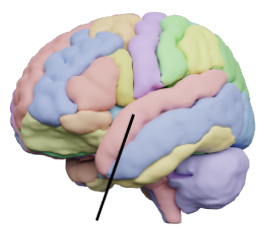

sup
The Atlas Concordance Problem

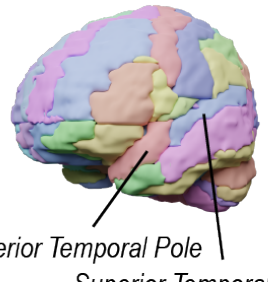

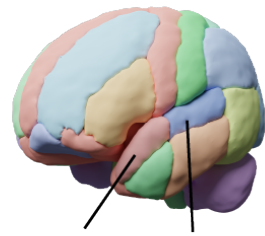

Anterior Posterior Temporal Pole Anterior

Superior Temporal Gyrus

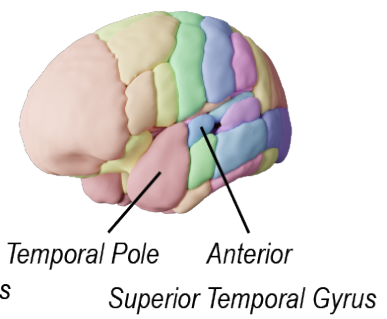

C Atlas choice alters connectivity; what is true connectivity?

\section{Cortically-bounded \\ Large or few parcels \\ Small or many parcels \\ Cortically-bounded parcels}

(e.g. DKT) axon
connections
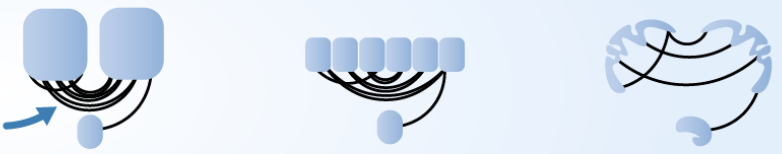

Subcortical (e.g. Harvard-Oxford)

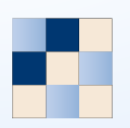

Resulting connectivity matrices

How does atlas choice affect study results? (specifically in quantifying structure-function correlation)
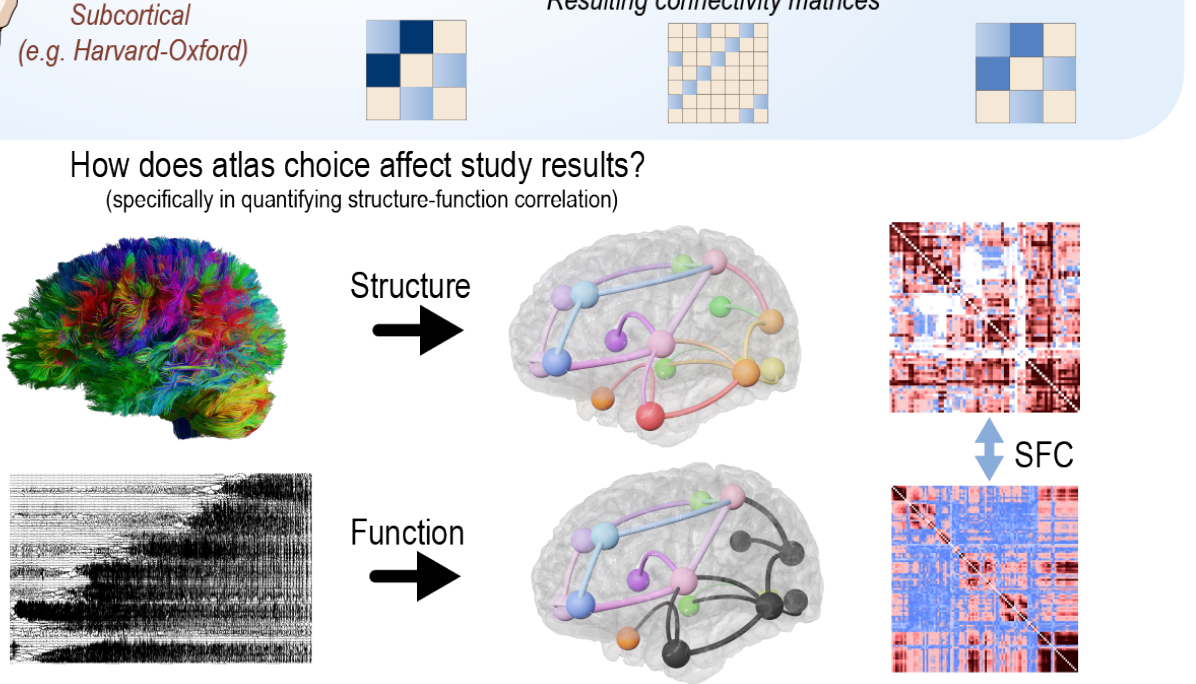

Fig. 1. Many brain atlases are available in the neuroscience literature. $\mid \mathbf{a}$, In common usage, an atlas refers to a "collection of maps" ${ }^{10}$ that defines geo-political borders at different scales. Although borders ${ }^{11}$ are usually consistent across atlases of the world, they are typically not consistent across atlases of the brain. Four separate atlases (left-to-right: CerebrA, AAL, Hammersmith, Harvard-Oxford) may define the superior temporal gyrus differently. The lack of consistency across these labels poses a problem for reproducibility in cognitive, systems, developmental, and clinical studies, as well as metanalyses describing the involvement of different regions of the brain in various diseases ${ }^{4}$. This challenge has been previously referred to as the Atlas Concordance Problem. b, Atlases can have varying features (see also Table 1). c, Thus, all current connectivity studies in neuroscience may not accurately reflect some fundamentally "true" architecture. For example, atlases with either large or small parcels may affect the structural connectivity matrices that are used to define the "true" network architecture of the brain, and subsequently that are used to test hypotheses or make predictions about the brain. d, When combined with white matter tracts reconstructed from diffusion MRI, atlases can be used to measure how different regions of the brain are structurally connected (i). Similarly, intracranial EEG (iEEG) implants can record neural activity to measure how different regions of the brain are functionally connected (ii). Technologies such as fMRI, MEG, and many others can also measure functional connectivity. The statistical similarity between structural and functional connectivity measurements can be calculated (e.g., structure-function correlation; SFC). Such estimates have been used to better understand the pathophysiology of disease. In this study, we evaluate how the varying atlases may alter the power to test a specific hypothesis about the brain's structure-function relationship in epilepsy.

when designing a neuroscience experiment. This framework is derived from historical foundations for assessing the validity and effectiveness of animal models ${ }^{25}$, network models ${ }^{26}$, and psychometric tests $^{27}$, which try to maximize the (1) descriptive, (2) explanatory, and (3) predictive validity ${ }^{26}$ of a model. Atlases are a tool for investigators to test for causality and 
bioRxiv preprint doi: https://doi.org/10.1101/2021.06.11.448063; this version posted October 7, 2021. The copyright holder for this preprint (which was not certified by peer review) is the author/funder, who has granted bioRxiv a license to display the preprint in perpetuity. It is made available under aCC-BY-NC 4.0 International license.

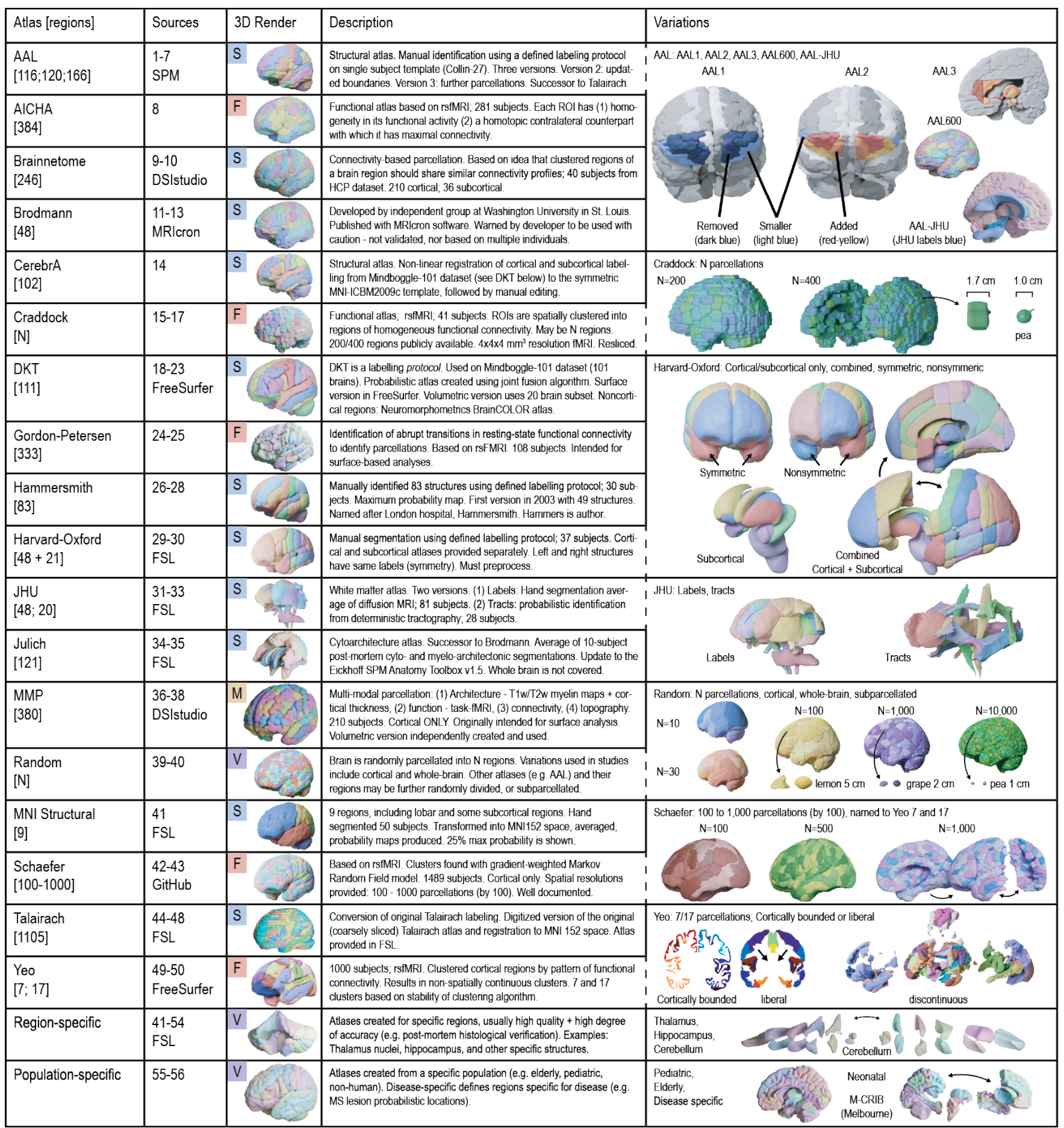

Table 1. Atlases. | Atlas sources are detailed in Table S1 and abbreviations are in the glossary. S: Structurally defined atlas; F: Functionally defined atlas; M: Multi-modally defined atlas; V: A variably defined atlas that may be structurally, functionally, or multi-modally defined; ROI: region of interest; HCP: Human connectome project dataset ${ }^{12}$; MS: multiple sclerosis.

62 to make predictions about the brain. Thus, this framework 63 incorporates a short discussion on explanatory modeling and 64 predictive modeling, each with different goals ("To Explain or 6 to Predict?" $\left.{ }^{15}\right)$. A one-size-fits-all approach may not exist for 66 selecting an atlas, nor should it ${ }^{28}$; while there is one Planet 7 Earth with a single atlas for a particular use (e.g., an atlas of the geo-political borders for a given point in time), there are many brains, with anatomical and functional variability across populations and species ${ }^{28}$. We hope our framework provides empirical guidance to neuroscience research utilizing the various atlases published over the last century. 

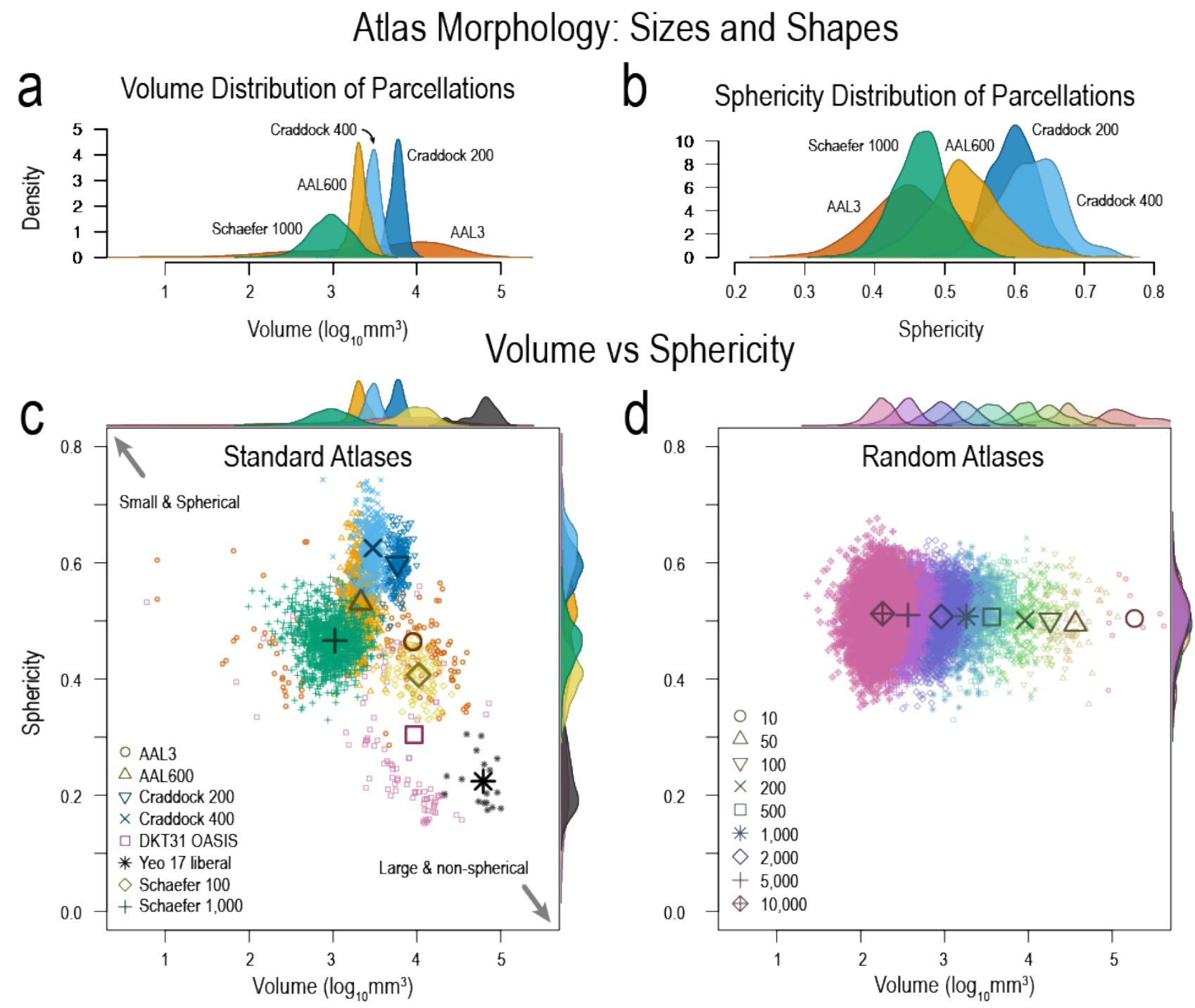

Fig. 2. Atlas morphology: sizes and shapes. | a, Volume distribution of atlas parcellations demonstrating the diversity of parcellation sizes. b, Parcellation sphericity distributions illustrating how the shapes of different parcellations may not be uniform. c, Volumes versus sphericity showing how some atlas parcellations may be small and spherical, while others may be large and non-spherical. This illustrates the non-uniformity in atlas parcellations. d, Volumes and sphericity of random atlases showing the uniformity of sphericity with changing volumes. Random atlases allow us to study (1) the effect of parcellation scale without the confound of shape effects and (2) the need for accurate anatomical boundaries to test a hypothesis about the structure-function relationship in the brain at seizure onset. Numbers in legend represent the number of parcellations for each random atlas. Remaining atlases are in Fig. S2.

\section{Results}

Clinical Data. Forty-one individuals (mean age $34 \pm 11 ; 16$ female) underwent High Angular Resolution Diffusion Imaging (HARDI), composed of thirteen controls (mean age $35 \pm 13$; 6 female) and twenty-eight drug-resistant epilepsy patients (mean age $34 \pm 11$; 12 female) evaluated for surgical treatment. Of the twenty-eight patients, twenty-four were implanted with stereoelectroencephalography (SEEG) and four with electrocorticography (ECoG). Ten SEEG patients (mean age $34 \pm 8$; 4 female) had clinical seizure annotations, and the first seizure from each patient (mean duration 81s) without artifacts was selected for SFC analyses. Patient and control demographics are included in Table S2.

Atlas Morphology: Sizes and Shapes. We hypothesized that atlas morphological properties, including size and shape (Fig 2), affect SFC. To test this hypothesis, we first quantified the distributions of parcellation sizes (Fig 2a) and shapes (Fig $2 \mathrm{~b}$ ) in various atlases. These results exemplify the diversity of atlas parcellation morphology. Fig 2c shows a comparison of individual parcellation volumes and sphericities. The remaining atlases are shown in Fig. S2. In contrast to standard 

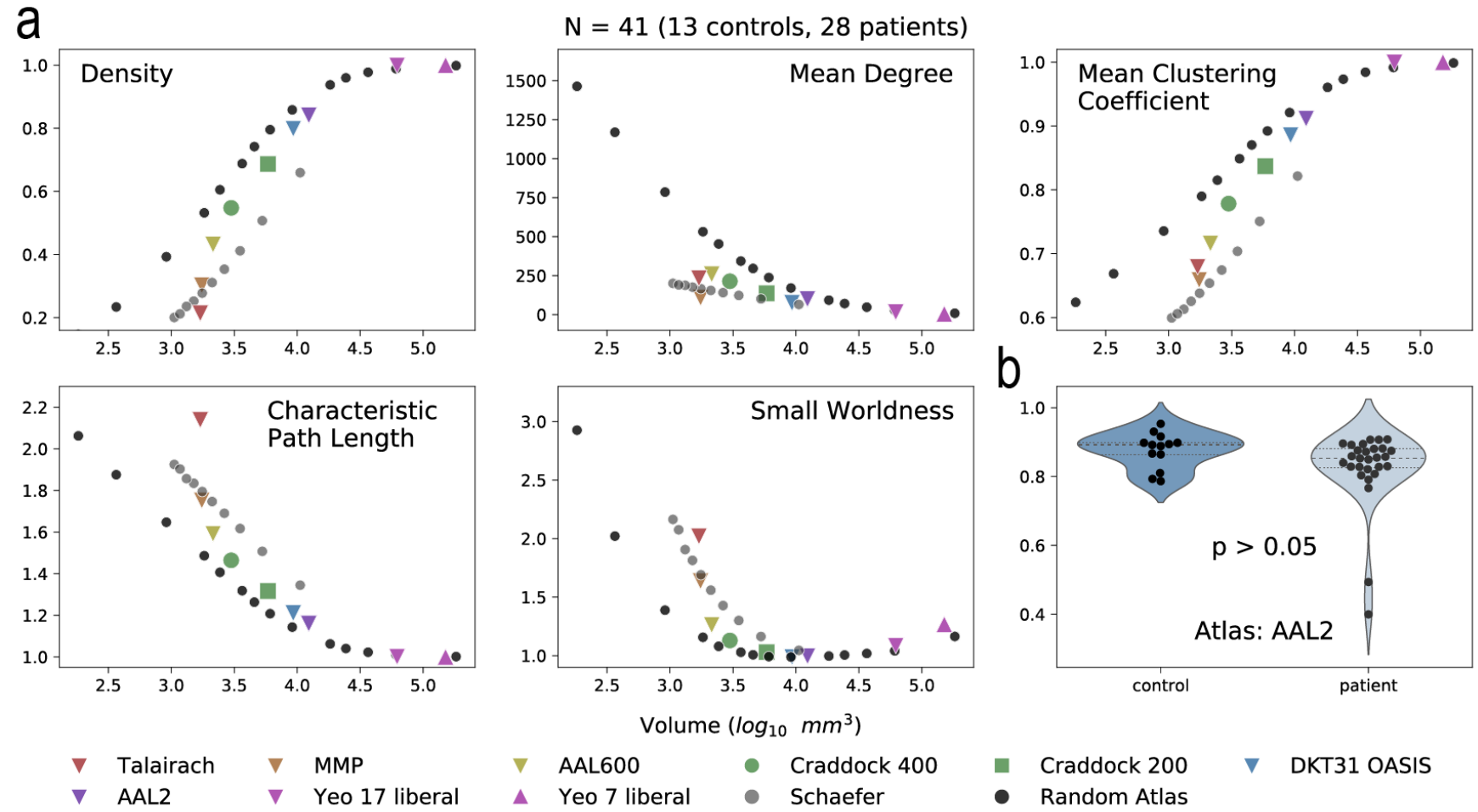

$\triangle \quad$ Yeo 7 liberal

- Schaefer

- Random Atlas

Fig. 3. Structural network differences between atlases. | a, Density, mean degree, mean clustering coefficient, characteristic path length, and small worldness were calculated for structural connectivity networks. A subset of atlases is shown. Remaining atlases studied are shown in Fig. S3. The average parcellation volume was calculated for each atlas and the corresponding network measure was graphed as the mean of all subjects $(\mathrm{N}=41 ; 13$ controls, 28 patients). b, Controls and patients were not significantly different in density for the AAL2 atlas (Mann-Whitney U test), illustrating that global structural network measures are similar between cohorts. However, specific edge-level connections between cohorts may be different, and characterizing these differences is out of the scope of this manuscript. Controls and patients were separated and shown in Fig. S4. Network measures using different threshold are shown in Fig. S5.

atlases, random atlases have constant sphericity with respect to volume size. Note that the distribution of parcellation shapes (i.e. sphericity) is similar across parcellation sizes in random atlases and their parcellations may not represent true anatomical or functional boundaries. Thus, random atlases allow us to study how parcellation scale affects network structure and SFC while keeping the effect of shape constant. Crucially, random atlases also allow us to explore if accurate and precise anatomical boundaries are essential in some experimental designs.

Varying atlases affect structural network topology. Although the morphology of atlas parcellations is diverse, we aimed to investigate how these morphological characteristics (particularly parcellation scale) affect structural network topology (Fig. 3). Networks are the basis upon which we compute SFC, and not necessarily morphological characteristics, therefore, we measured how network density, mean degree, characteristic path length, mean clustering coefficient, and small worldness change as a function of parcellation scale (Fig. 3a). We found that the change in these network measures are congruent between standard and random atlases and previous studies ${ }^{29}$. We also show that mean density, a global network measure, is similar between our control $(\mathrm{N}=13)$ and patient $(\mathrm{N}=28)$ cohorts (Fig. 3b).

Varying atlases affect SFC: single subject. Fig. 4 illustrates an overview of how SFC is calculated. Structure is measured with high angular resolution diffusion imaging (HARDI) and function is measured with SEEG electrode contacts. Structural connectivity matrices are generated based on the atlas chosen (Fig. 4a) and functional connectivity matrices are generated based on broadband $(1-127 \mathrm{~Hz})$ cross-correlation of neural activity between the electrode contacts in widows of time (Fig. 4b, see Methods section on "Functional Connectivity Network Generation"). Thus, the structural network is static while the functional network is computed across time. The connectivity matrices shown are example data from a single patient, sub-patient07. Functional connectivity matrices are shown for 6 hours before seizure onset, 90 seconds before seizure onset $(\mathrm{t}=-90), 40$ seconds after seizure onset $(\mathrm{t}=$ 40), 88 seconds after seizure onset (seizure duration $=89$ seconds), and 180 seconds after seizure onset (91 seconds after seizure termination). Each functional connectivity matrix time window was correlated to each structural connectivity matrix, yielding a SFC at each time window (Fig. 4c). Each point represents the structural edge weight between two brain regions and their corresponding functional connectivity edge 


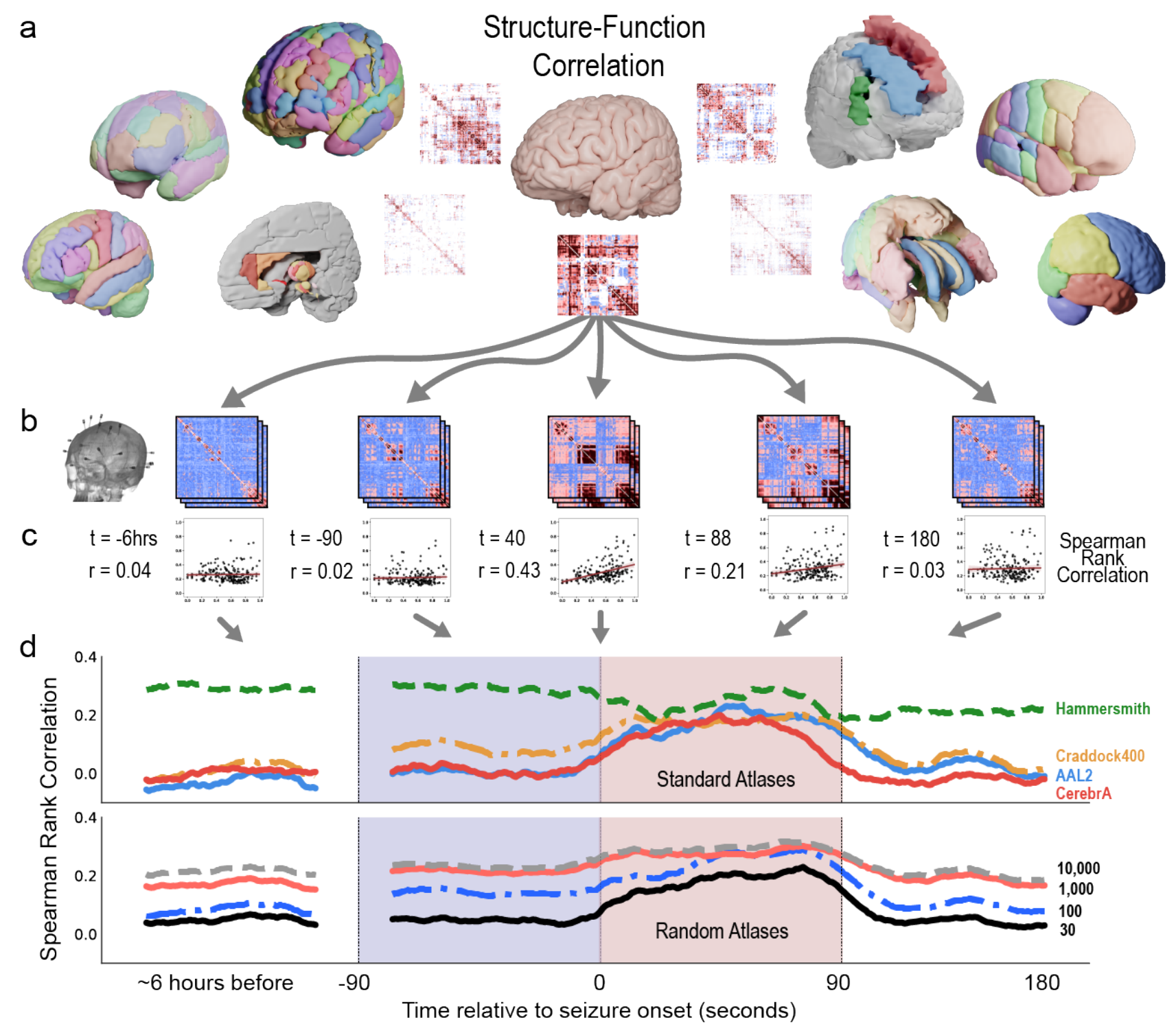

Fig. 4. Structure-Function correlation in a single patient using different atlases. | a, Example atlases and structural connectivity matrices. b, Functional connectivity matrices are computed from SEEG recordings during the interictal, preictal, ictal, and postictal periods. During each period, the SEEG data is binned into non-overlapping windows (the vertically stacked matrices) to create time varying representations of functional connectivity. Broadband cross correlation matrices are shown for sub-patient07 at 6 hours before seizure onset, 90 seconds before seizure onset, 40 seconds after seizure onset $(\mathrm{t}=40)$, 88 seconds after seizure onset (seizure duration $=89$ seconds), and 180 seconds after seizure onset (or 91 seconds after seizure termination). c, Each functional connectivity matrix is correlated to a structural connectivity matrix of a given atlas. Spearman Rank Correlation is measured between all time points and all atlases for each patient. Lines of best fit are for visualization purposes only. d, SFC is graphed at each time point for four example standard atlases (Hammersmith, Craddock400, AAL2, and CerebrA), and four example random atlases $(30,100,1 \mathrm{k}$, and 10k parcellations). SFC increases during seizure state for some standard atlases (Craddock 400, AAL2, and CerebrA atlases). This result follows previous SFC publications with $\mathrm{ECoG}^{13,14}$. However, SFC does not increase for the Hammersmith atlas. These findings highlight that the power to detect a change in the structure-function correlation at seizure onset, and thus the ability to probe the hypothesis that seizure activity is correlated to brain structure, may be reduced using some atlases. The use of different atlases may contradict previous studies.

weight in broadband cross-correlation. A line of best fit is shown for visualization, and $\mathrm{r}$ values represent Spearman rank correlation for that time point. SFC was graphed for all time points during the interictal, preictal, ictal, and postictal periods for this patient in Fig. 4d.

Four example standard and random atlases are graphed. We show that SFC increases during the ictal state for many atlases (CerebrA, AAL2, Craddock 400), but not all atlases 


\section{Average of all seizures (broadband cross correlation)}
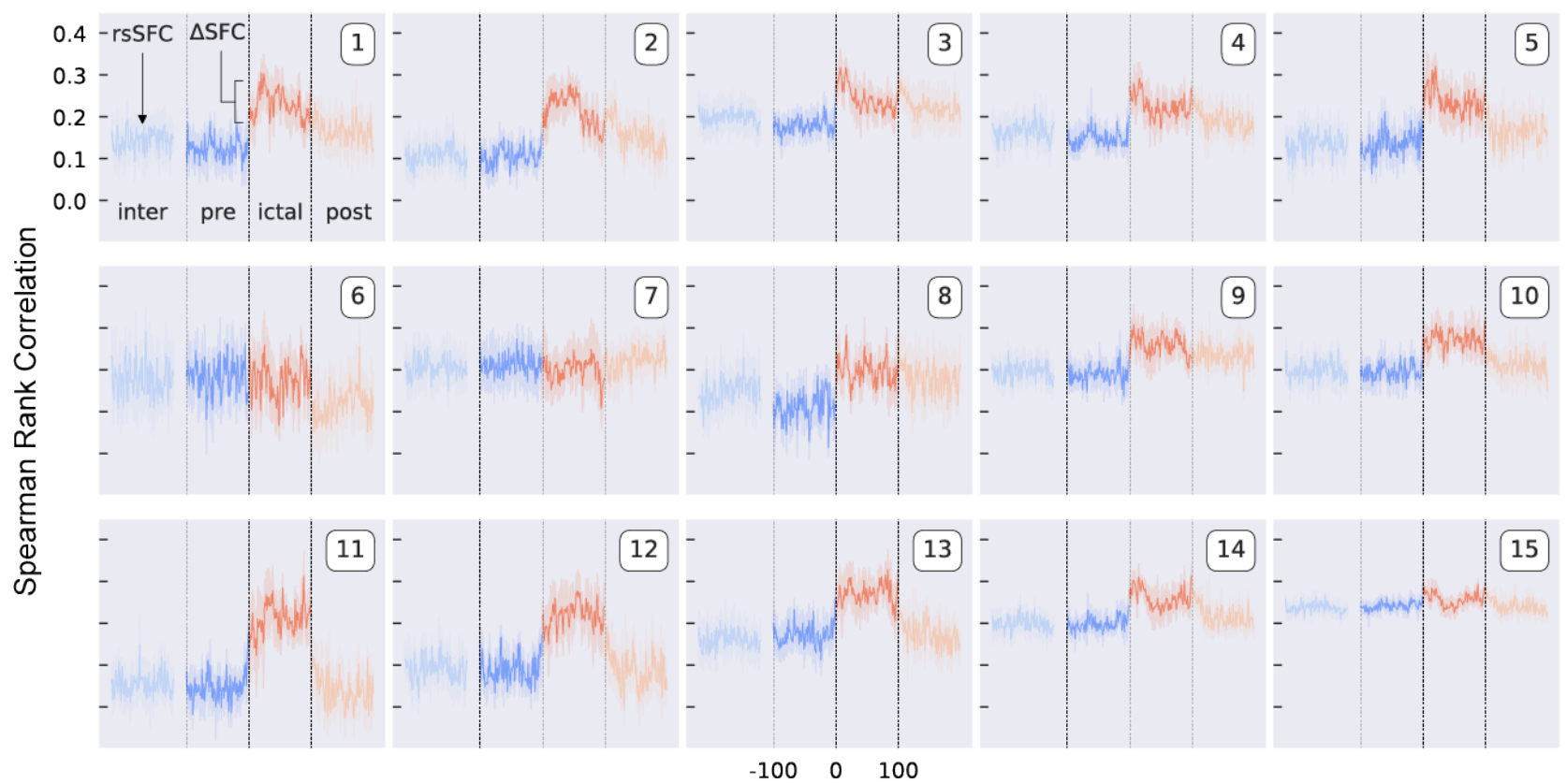

Time (normalized to 100; seizure start $=0$ )
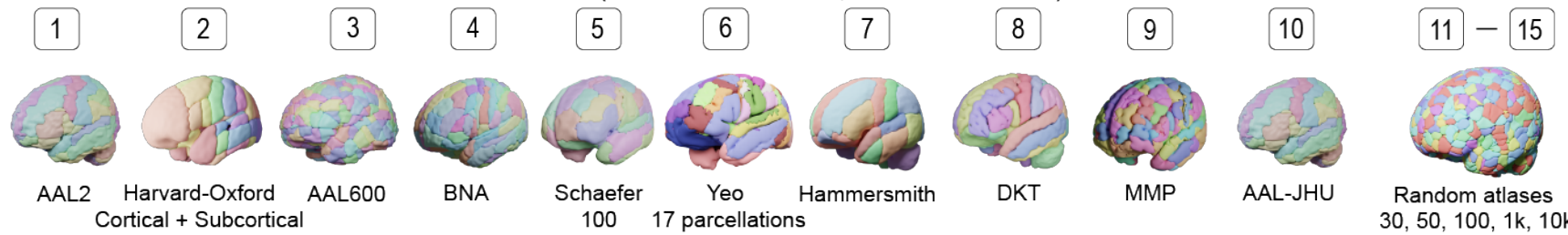

Fig. 5. Structure-Function Correlation in multiple patients using different atlases. | SFC for ten standard atlases and five random atlases using SEEG broadband cross-correlation matrices averaged across all patients with clinically annotated seizures $(\mathrm{N}=10)$. Resting state SFC (rsSFC) is the SFC during the interictal period. The change from preictal to ictal SFC is $\triangle$ SFC. SFC was similarly calculated for random atlases and shows that rsSFC and $\triangle$ SFC may change with parcellation scale. These findings may be concerning given that the inherent structure-function relationship in the brain is not necessarily changing at resting state, but its measurement is greatly affected by atlas choice alone.

(Hammersmith). The increase in SFC during seizures follows previous SFC studies using ECoG ${ }^{13,14}$. Similarly, SFC increases for a subset of random whole-brain atlases. While parcellation scale may affect SFC, it is not the only feature affecting SFC - the Hammersmith and AAL2 atlases have similar parcellation scales yet diverging neuroanatomical properties and SFC dynamics. These findings highlight inference from one type of atlas may suggest that seizure activity is not correlated to brain structure, contradicting previous studies ${ }^{13}$

Varying atlases affect SFC: multiple subjects. Fig. 5 shows SFC for ten standard atlases and five random atlases using SEEG broadband cross-correlation metrics averaged across all patients with clinically annotated seizures $(\mathrm{N}=10)$. Functional connectivity measurements were also calculated for coherence, zero time-lag Pearson, and Spearman rank correlations across multiple frequency bands. They are included in the freely available, curated, and opensource dataset (see Methodology section on "Data Availability and Reproducibility" for links); however, they were not used in directed hypothesis testing about specific frequency bands nor about other functional measurements in the present study. The AAL2 atlas shows a statistically significant increase in SFC from preictal to ictal periods ( $\mathrm{p}<0.05$ by Wilcoxon signed rank test after Bonferroni correction for 52 tests). This change from preictal to ictal SFC is denoted $\triangle \mathrm{SFC}$. Using the AAL2 atlas, this finding supports the hypothesis that seizure activity propagates and spreads via axon tracts making up the underlying structural connectivity of the brain ${ }^{13,14}$. SFC was similarly calculated for random whole-brain atlases. A notable finding is that during the interictal period, resting state SFC (rsSFC) increases at larger number of parcellations (i.e. smaller parcellation volumes). We show that rsSFC is observably affected by parcellation scale when inspecting the random atlases in Fig. 5 (bottom row). These findings may be concerning given that the inherent structure-function relationship in the brain is not necessarily changing at resting state, but its measurement is greatly affected by atlas choice alone. 

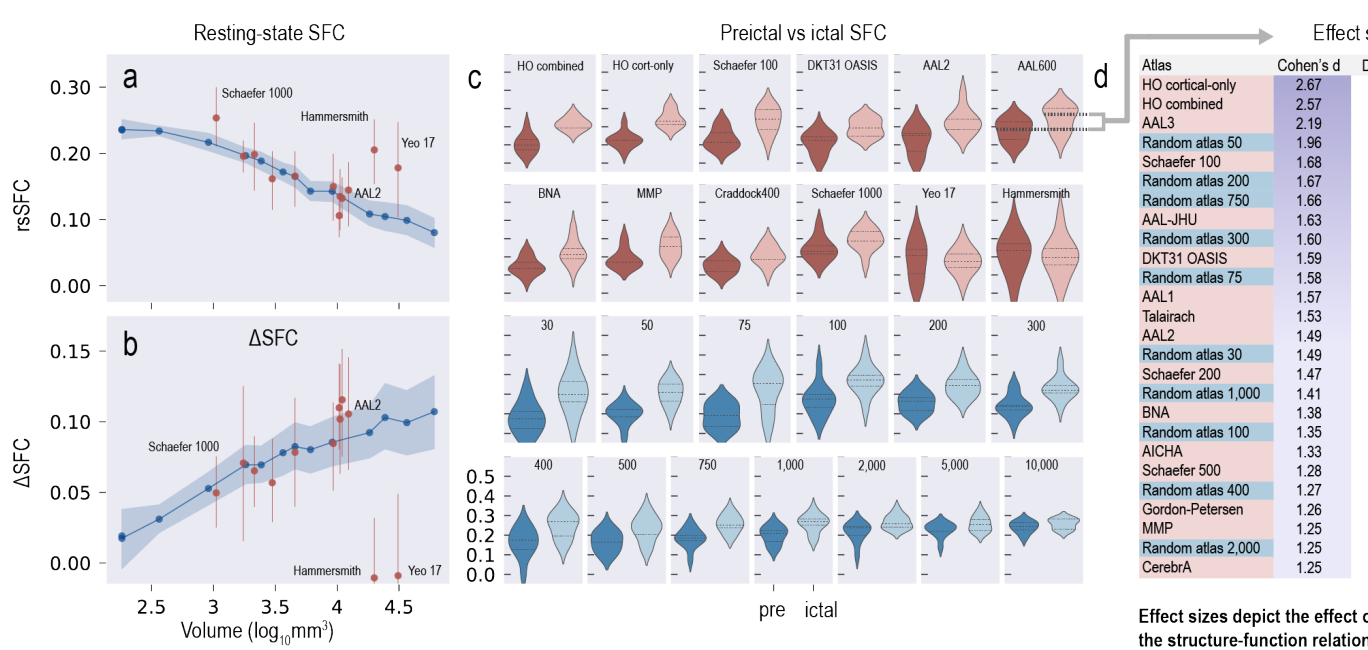

Effect sizes of all atlases

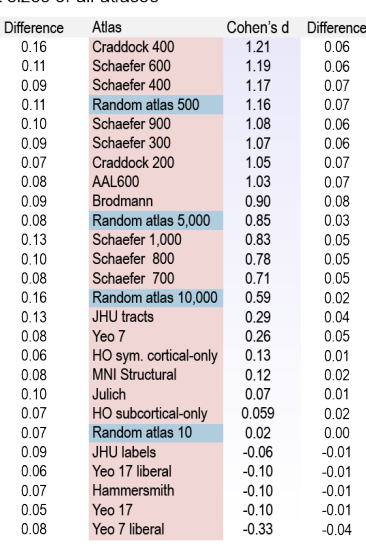

Effect sizes depict the effect of atlas choice and atlas features on quantifying the structure-function relationship of the brain in this study design

Fig. 6. The power to test a hypothesis about epilepsy pathophysiology changes depending on atlas choice | a, Resting state SFC (rsSFC) decreases with larger parcellation volumes (moving left to right). Random atlases are shown in blue, and select standard atlases are shown in red. Points represent the average across all patients, and bands represent $95 \%$ confidence intervals. b, $\triangle \mathrm{SFC}$ increases with larger parcellation volume (moving left to right). Broadly, $\mid$ DeltaSFC may be interpreted as the change in SFC with respect to disease (e.g. a seizure) and non-disease states, and this change has been used to characterize and make inferences on many neurological diseases. These results exemplify that parcellations that are either too coarse (large volumes) or too fine (small volumes) may not adequately capture the underlying SFC of the brain or its dynamics with relation to a neurological disease. c, A subset of atlases show a difference in preictal and ictal SFC. d, The effect size between preictal and ictal SFC is calculated for all 52 atlases used in this study. Many atlases commonly used in the neuroscience literature have comparable effect sizes to random atlases. The standard atlases with the greatest effect size (and thus power) are the Harvard-Oxford and AAL3 atlases. These atlases outperform many random atlases (where anatomical boundaries are not followed) and may indicate that their parcellation scheme captures the structure-function relationship in the brain at seizure onset with DTI and iEEG.

Varying atlases affect resting state SFC and $\triangle$ SFC. Resting state SFC (rsSFC) and the change in SFC ( $\triangle \mathrm{SFC})$ from preictal to ictal periods are affected by parcellation scale (Fig. 6). Fig. 6a shows how rsSFC decreases with larger average parcellation volumes (moving left to right). A large average parcellation volume for a given atlas generally means there is a fewer number of total parcellations (e.g. the MNI structural atlas has a large average parcellation volume given only nine parcellations). In contrast, Fig. $6 \mathrm{~b}$ shows $\triangle \mathrm{SFC}$ increases with larger parcellation volumes (moving left to right). Broadly, $\triangle \mathrm{SFC}$ may be interpreted as the change in SFC with respect to a disease (e.g. a seizure) and non-disease states. This change metric has been used to characterize and make inferences in many neurological disorders ${ }^{30,31}$. Only a subset of atlases show a change in SFC at seizure onset (Fig. 6c). These results exemplify that either overly coarse or fine parcellations may not adequately capture the underlying SFC of the brain or its dynamics with relation to a neurological disease.

Atlas choice affects the power to test a hypothesis. The effect size between preictal and ictal SFC is calculated for all 52 atlases used in this study (Fig. 6d). Cohen's d and the difference between the mean ictal and mean preictal SFC are shown. Atlases are ordered by Cohen's d.

We found that different atlases may alter the power to test the hypothesis about epilepsy pathophysiology that seizures propagate through the underlying structural tracts of the brain, measured with diffusion MRI. This hypothesis has been previously supported in prior studies ${ }^{13,14,23,24}$

Many atlases commonly used in the neuroscience literature have comparable effect sizes to random atlases (where anatomical boundaries are not followed). The standard atlases with the greatest effect size (and thus power, given equal significance levels and sample sizes) are the Harvard-Oxford and AAL3 atlases. These atlases outperform many random atlases and may indicate that their parcellations may adequately capture the structure-function relationship in the brain. These atlases may capture the "true" structural network architecture (see Fig. 1c) because these network architectures better differentiate and are more correlated to functional changes seen at seizure onset.

\section{Discussion}

In this study, we performed an extensive evaluation of the available structural, functional, random, and multi-modal atlases in the neuroscience literature (Table 1). We detailed morphological (Fig. 2) and network (Fig. 3) differences between these atlases. We showed the effect of atlas choice on the measurement of structure-function correlation (SFC) in epilepsy patients (Fig. 4 and Fig. 5). We also showed how various atlases may affect the power to test a hypothesis about seizure propagation (Fig. 6). This work has implications for investigators because the ability to test hypotheses and make predictions about the brain's function may depend on atlas choice. In light of our study of the structure-function rela- 
bioRxiv preprint doi: https://doi.org/10.1101/2021.06.11.448063; this version posted October 7, 2021. The copyright holder for this preprint (which was not certified by peer review) is the author/funder, who has granted bioRxiv a license to display the preprint in perpetuity. It is made available under aCC-BY-NC 4.0 International license.

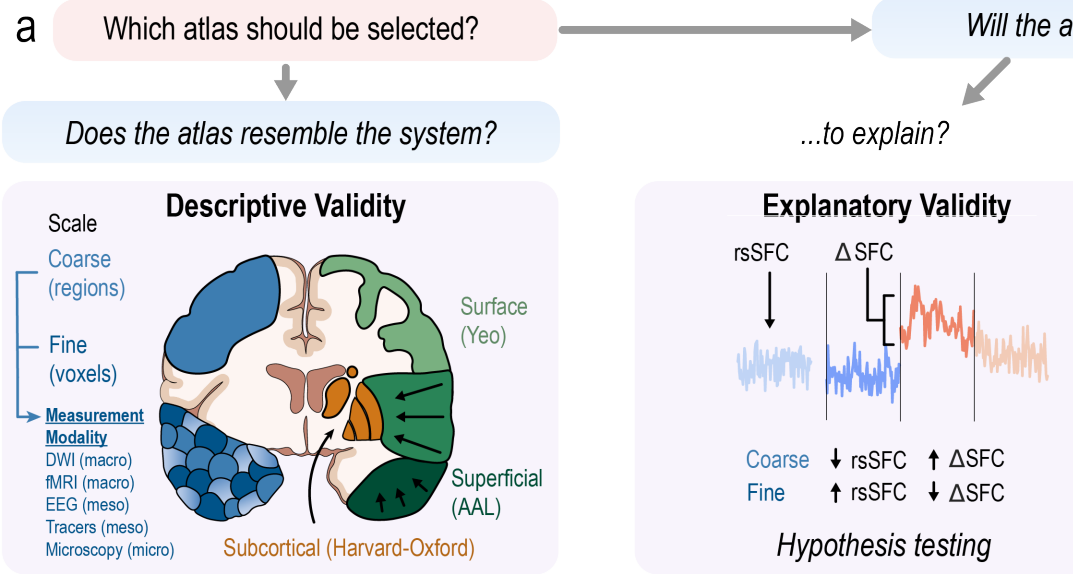

\section{b Atlas Features $\downarrow$}
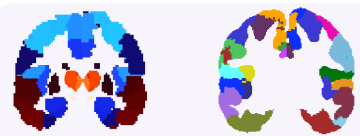

Structural

Functional

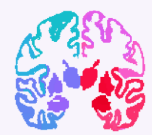

Grayordinate

Cortical

Only
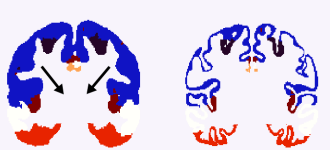

Liberal vs Conservative

C Questions to consider

Do I need specific anatomical regions (e.g. hippocampus)? Am I testing hypotheses or making predictions about specific regions?

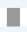

Are there any consequences from leaving out brain regions (white matter, cerebellum, subcortical structures)?

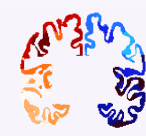

Multimodal

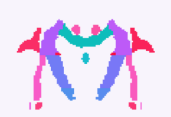

Volumetric

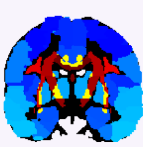

Combined White Matter
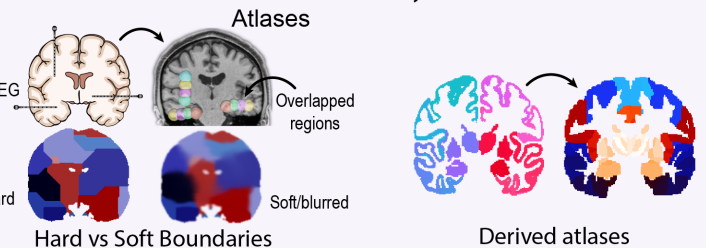

Derived atlases

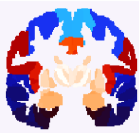

Connectivity

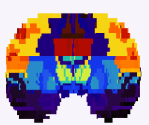

Whole Brain

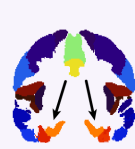

Do I need accurate parcellations (how does accuracy affect the power of my study)? .

Am I studying small structures where precise localization is needed, even in the setting of variability across subjects?

Should I use a subject-specific atlas where labels are created from a trained classifier?

What are the computational costs, resources, time, and personnel training needed? Can another atlas be used instead (and may have similar power to test hypotheses)?
Can I use random atlases instead and - permute the results over many generated random atlases?

Should I use more than one atlas?

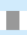

Am I making claims about specific brain regions and are there other atlases with similar parcellations to check the robustness of results?

Do I need a volumetric or surface-based atlas? What use was an atlases intended for? How was it developed?
... or to predict?

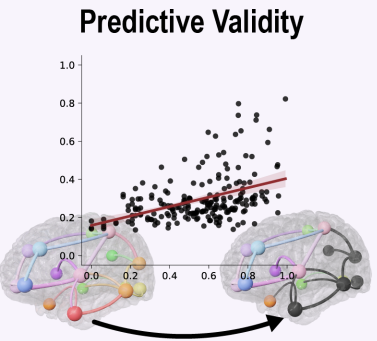

Predict new or future observations

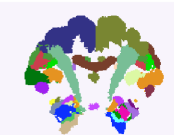

Cytoarchitecture/ Molecular/Genetic

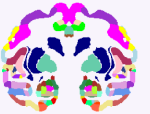

Non-Human (Macaque)

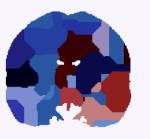

Random

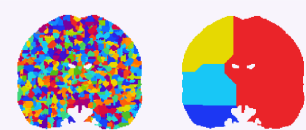

voxels $\rightarrow$ lobes (many/small) (few/large)

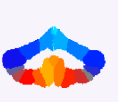

Regional (Cerebellum)

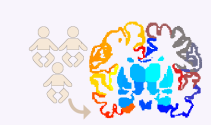

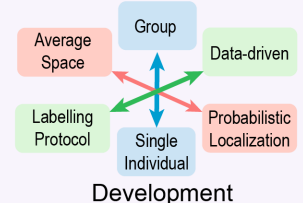

If I use a (custom) atlas, how will that affect replicability and meta-analyses studying results across neuroscience?

\section{-}

Should I use a study-specific atlas tailored to the participants in the study? How would this atlas affect replicability and translation?

How would parcellation scale

affect my results?

Do I need a structurally-, functionallymultimodally-, or randomly-defined atlas?

Fig. 7. A Framework for brain atlases. | a, An important question in the neuroscience community is: Which atlas should be chosen for a study? We propose a framework that helps select an atlas in the context of its descriptive, explanatory, and predictive validity. Descriptive validity of an atlas addresses that its features appropriately resembles the experimental system. An atlas is a tool to tackle a wide variety of problems in neuroscience. It may be used as part of a methodology to explain causality (explanatory validity), or it may be used to make predictions (predictive validity). These two goals are distinct, and the differences between explanation and prediction "must be understood for progressing scientific knowledge" 15 . In the context of building scientific models, ones with high explanatory power may not be of high predictive power ${ }^{15}$. Atlases are also part of the scientific methodology to explain how the brain functions or to make predictions. These aspects (to explain or to predict) should be considered when selecting an atlas and are detailed further in the main text. b, Non-mutually exclusive atlas features related to descriptive validity. Across neuroscience, many atlases with specific features tailored for a study have been developed. c, A list of questions to consider when choosing an atlas. Related questions are connected by gray lines. 
bioRxiv preprint doi: https://doi.org/10.1101/2021.06.11.448063; this version posted October 7, 2021. The copyright holder for this preprint (which was not certified by peer review) is the author/funder, who has granted bioRxiv a license to display the preprint in perpetuity. It is made available under aCC-BY-NC 4.0 International license.

tionship in the brain using an extensive list of available brain atlases, we propose a general framework below for evaluating and selecting an atlas (Fig. 7).

A Framework for Brain Atlases. Various publications have highlighted the Atlas Concordance Problem ${ }^{2-4,9}$, curated several atlases in freely accessible databases ${ }^{32,33}$, and made arguments for why specific atlas features (Fig. 7b) may be superior in certain situations ${ }^{21,28,34-38}$. There have been great efforts to publish accurate and precise parcellations as seen with an exponential rise in atlas-related publications over the last three decades (Fig. S7). However, none have found a general solution to the underlying problem: Does atlas choice matter?

We provide a framework that allows us to determine if the choice of an atlas is appropriate in the context of its (1) descriptive, (2) explanatory, and (3) predictive validity ${ }^{26}$. This framework is borrowed from the logic for assessing network models ${ }^{26}$, animal models, ${ }^{25,39}$, and psychometric tests ${ }^{27,40}$, where assessment of these models with standard statistical model-selection methods is particularly challenging.

Descriptive validity of an atlas refers to an atlas that appropriately resembles the system in which we work. In other words, it has "face value" ${ }^{25}$. An atlas should include features (Fig. 7b) relevant to the study (e.g., parcellations containing subcortical structures relevant to epilepsy). Importantly, descriptive validity of an atlas also relates to the modality scale we use to measure the brain - for example, DWI and fMRI at the macroscale ${ }^{41}$, iEEG and tracers at the meso scale ${ }^{42}$, and microscopy at the microscale ${ }^{43}$. It is important to select a parcellation scale that resembles the measurement modality resolution (Fig. 6a). When correlating DWI with iEEG in our study at larger parcellation sizes, we lose our ability to discern precise anatomical locations that are structurally and functionally related (Fig. 6b). Similarly at smaller parcellation sizes (tending to voxel resolution), we may not capture the "true" structural network architecture (Fig. 1c), and thus we lose our ability to capture structure-function relationship changes at seizure onset.

An atlas is a tool to tackle a wide variety of problems in neuroscience. It may be part of a methodology to explain causality (explanatory validity) or it may be part of a methodology to make predictions (predictive validity). These two goals are distinct, and the differences between explanation and prediction "must be understood for progressing scientific knowledge" as described in "To Explain or Predict?" by Shmueli, $2010^{15}$. In the context of building scientific models, a model with a high explanatory ability may not have a high predictive ability.

Similar to models, atlases are also part of a scientific methodology to (1) explain how the brain functions or (2) predict new observations. An atlas may be suitable for hypothesis testing, for example, because it includes subcortical structures like the hippocampus (also high descriptive validity) to support a hypothesis about seizure propagation through subcortical structures. Intuitively, without subcortical structures, it would be impossible to test hypotheses about subcortical structures. Less intuitively, explanatory validity of an atlas may also relate to the power to test hypotheses, which we show in our study. Some atlases may not be suitable for scientific inquiry because they provide little statistical power to detect differences in disease states, for example, to detect changes in SFC at seizure onset (Fig. 6b). Power may depend on the accuracy of anatomical boundaries, or in our study, other atlas features such as parcellation scale and configuration (Fig. 6d). For example, the Harvard-Oxford and AAL3 atlases have similar parcellation configurations and similar power.

Some atlases may or may not be not be suitable for making predictions about new or future observations about the brain. For example, many network properties change with atlas choice (Fig. 3), and thus it is reasonable to suspect model prediction outputs may change with respect to the atlas used to build and train such models. Importantly, the exclusion of some anatomical structures, like white matter or the cerebellum in some atlases, may affect the training data used to build predictive models. In our study, a translational goal is to predict functional seizure activity from structural data SEEG records activity from both gray matter and white matter; however, recent studies have shown that white matter functional recordings may provide different information than gray matter ${ }^{44-47}$. Thus, excluding some anatomical labels may affect model predictions. Another example is the use of network models to predict spread, such as $\alpha$-synuclein across the brain connectome ${ }^{48}$. Without the incorporation of all brain structures related to $\alpha$-synuclein spread, models to predict and monitor spread may be inaccurate. Note that the cerebellum, which is involved in many functional tasks, is excluded in many atlases.

Are accurate anatomical or functional parcellations needed? During the course of conducting this study, and while undergoing revisions, other atlases with more accurate or relevant parcellations to the study's population were published in different areas of neuroscience ${ }^{49-51}$. Here, we cautiously propose a question: Are efforts to publish more atlases created with different algorithms or slightly modified parcellations from existing atlases providing any advantages over already existing atlases? Naturally, accurate and precise parcellations are needed when probing specific hypotheses about exact structures that depend on accurate segmentation of such structures (particularly at the sub-field or cellular level; see next section below); however, few studies compare an atlas to a null atlas (one with randomly generated parcellations).

In this study, we show that random atlases provide similar power to detect differences in SFC between preictal and ictal states (Fig. 6d). Indeed, it is difficult or nearly impossible to evaluate a newly proposed atlas, given that the performance metrics to evaluate an atlas may be infinite (given infinite experimental designs). Only one such metric, SFC, was used in this study. But given new deep learning methods and other computationally expensive methods using trained classifiers for segmentation, existing atlases may be adequate for labs with limited funding resources, trained personnel, and access to GPUs. These labs may still be capable of answering important questions in neuroscience.

Bridging atlases from the cellular scale to the neuroimaging scale. Precise mapping of the brain that bridges molecules and cells to the macroscale level of gross neuroanatomy is an essential step towards understanding the hierarchical organization and unifying mechanistic framework of brain function across scales ${ }^{51-53}$. Recently, multiple research groups and institutions have begun mapping in detail the molecular and cytoarchitecture organization of the human brain ${ }^{54}$ and of 
other species ${ }^{53,55}$. Bridging the gap is outside the scope of this study. However, atlases defined by human cytoarchitecture were included in this study. Our results begin to probe how macroscale structural networks connecting cytoarchitecturallydefined regions relate to the function of those regions during seizures.

Which atlas should be used for my study?. The framework provided above may be abstract to some readers; therefore we also provide a concrete list of questions to consider when choosing an atlas (Fig. 7c) for a neuroimaging study. We hope our discussion here helps avoid testing large numbers of atlases at one extreme (and thus giving rise to multiple comparison problems) and supports the current standard of using a single atlas in the main text of a publication and, if deemed necessary, providing supplementary results using a different atlas (preferably one with different features). In our study, most atlases give similar effect sizes (Fig. 6d) and would yield the same results. In the case where different atlases yield radically different results (rather than one atlas being under-powered), we suggest working though the framework and questions posed in Fig. 7c. Perhaps one atlas used was developed for another purpose. In Fig. 6d, many atlases intended for surface-based (e.g., Yeo) or white matter-only analyses (e.g., JHU) give contradictory results to most other atlases. In this case, it is feasible that a chosen atlas is missing important structures at an optimal resolution to test a hypothesis. However, most atlases yield similar results, only with different power.

Limitations. Our study is not without limitations. A major limitation is that we did not evaluate atlases in a diverse set of experimental systems, but rather limited our analysis to a contemporary topic in epilepsy using SEEG implantations and to a study of the structure-function of the brain, potentially appealing to a wider audience. The question we were trying to answer ("Which atlas should we use?") is a difficult problem to solve, given that it would be impossible to evaluate all atlases in all experimental designs. We attempted to generalize a framework given our findings after an extensive search for, and curation of, available neuroimaging atlases.

We also did not perform a feature selection analysis posthoc to maximize $\triangle \mathrm{SFC}$ at seizure onset; rather, we performed a comprehensive evaluation of many atlases to set a general framework and describe the nuances between the different atlases and their features. Ideally in our study, we required a whole-brain, volumetric atlas that covered the implanted SEEG electrode contacts. No such atlas existed. We opted for combining different atlases or developing randomly parcellated atlases used in previous publications ${ }^{29,56}$. However, no general framework existed to determine which atlas should be used or clearly outlined the feature space of these atlases. We had no formal basis for how changing an atlas could change our results and eventual goal for translating network models to better treat epilepsy patients.

Another limitation, we assume a change in SFC supports the hypothesis that seizures harness the underlying structural connectome of the brain (along with support from prior literature $^{13,14,57}$ ). We may be biasing our results to select an atlas that maximizes $\triangle \mathrm{SFC}$. However, we wish to select a methodology that allows us to measure any change in brain state that accompanies seizure onset (explanatory validity), permitting us to probe epilepsy biology and understand the processes that govern seizure spread.

An additional limitation concerns the effect of parcellation volume on SFC. In probing this effect across our random atlases and atlases used in the literature, we did not perform controlled experiments to separate the effects of parcellation size from parcellation $\mathrm{N}$ (number of parcellations). A future experiment could fix the number of parcellations while changing parcellation volume (or vice versa). This would allow us to test whether parcellation volume or $\mathrm{N}$ drives changes in SFC. However, this was outside the scope of our study.

Our goal was to highlight the importance of selecting an appropriate atlas from an array of possibilities, using a datadriven, validated experimental paradigm ${ }^{13}$. We acknowledge new studies that show that streamline counts may not completely reflect the underlying diffusion data ${ }^{58}$; however, comparing such techniques were outside the scope and goal of our focused study. We also note that few patients had lesions on imaging. Misalignment due to non-linear distortion may add noise to our data; however, few patients had lesions. Our study was not conducted to necessarily make the claim that SFC changes exist in the brain at seizure onset, but rather to show how varying atlases may change SFC.

Finally, our analysis relies on the assumption that an atlas approach must be used to quantify SFC and does not consider an atlas-agnostic approach nor if such an approach is appropriate. To study SFC using networks, both structural and functional networks must have nodes representing the same entity - neuroanatomical structures. The atlases defining anatomical structures (whether they are functionally, histologically, genetically, procedurally, multi-modally, or randomly defined) are the link between structural connectivity and functional connectivity measurements of the brain. To study SFC, we must rely on the neuroanatomical structures defined by an atlas, then localize electrodes to these regions and correlate the structural measurements (e.g., streamlines, fractional anisotropy, mean diffusivity) with functional measurements (e.g., cross-correlation, coherence, mutual information). Fundamentally, we are defining the nodes of the brain in advance, which can alter our results; a more comprehensive discussion on defining the nodes of the brain are in Fornito et al., 2016 and Bijsterbosh et al., $2017^{42,59}$.

Conclusion. The publication of atlases and their distribution across neuroimaging software platforms has risen exponentially over the last three decades. We simulate a study in which a researcher is blind to the development or features of an atlas and chooses one based on availability in common neuroimaging pipelines and software (e.g., Freesurfer, DSI studio, FSL, SPM, QSIprep, fMRIprep, MRIcron, ANTs, and others). Our study illustrates the critical need to evaluate the reproducibility of neuroscience research using atlases published alongside tools and analysis pipelines already established in the neuroscience community. Please see our GitHub for the atlases curated in this study along with their direct primary sources listed in Table S1. 
bioRxiv preprint doi: https://doi.org/10.1101/2021.06.11.448063; this version posted October 7, 2021. The copyright holder for this preprint (which was not certified by peer review) is the author/funder, who has granted bioRxiv a license to display the preprint in perpetuity. It is made available under aCC-BY-NC 4.0 International license.

\section{References}

[1] Klein, A. \& Tourville, J. 101 labeled brain images and a consistent human cortical labeling protocol. Frontiers in Neuroscience 6 (2012).

[2] Mandal, P. K., Mahajan, R. \& Dinov, I. D. Structural brain atlases: design, rationale, and applications in normal and pathological cohorts. Journal of Alzheimer's disease : JAD $\mathbf{3 1}$ Suppl 3, S169-88 (2012).

[3] Dickie, D. et al. Whole brain magnetic resonance image atlases: A systematic review of existing atlases and caveats for use in population imaging. Front Neuroinform 11, 1 (2017).

[4] Bohland, J. W., Bokil, H., Allen, C. B. \& Mitra, P. P. The brain atlas concordance problem: Quantitative comparison of anatomical parcellations. PLoS ONE 4, e7200 (2009).

[5] Beal, D. S. et al. The trajectory of gray matter development in broca's area is abnormal in people who stutter. Frontiers in Human Neuroscience 9 (2015).

[6] Van Horn, J. D. et al. Mapping connectivity damage in the case of phineas gage. PLOS ONE 7, e37454 (2012).

[7] Barker, F. G. Phineas among the phrenologists: the american crowbar case and nineteenthcentury theories of cerebral localization. Journal of Neurosurgery 82, 672-682 (1995).

[8] Mazziotta, J. et al. A probabilistic atlas and reference system for the human brain: International consortium for brain mapping (icbm). Philosophical Transactions of the Royal Society of London. Series B: Biological Sciences 356, 1293-1322 (2001).

[9] Evans, A. C., Janke, A. L., Collins, D. L. \& Baillet, S. Brain templates and atlases. Neurolmage 62, 911-922 (2012).

[10] Society, N. G. atlas. National Geographic Society (2011).

[11] Society, N. G. border. National Geographic Society (2011).

[12] Van Essen, D. C. et al. The wu-minn human connectome project: an overview. Neurolmage 80, 62-79 (2013).

[13] Shah, P. et al. Characterizing the role of the structural connectome in seizure dynamics. Brain a journal of neurology (2019).

[14] Ashourvan, A. et al. Pairwise maximum entropy model explains the role of white matter structure in shaping emergent co-activation states. Commun Biol 4 (2021).

[15] Shmueli, G. To explain or to predict. Statistical Science 25 (2010).

Makris, N. et al. Decreased volume of left and total anterior insular lobule in schizophrenia. Schizophrenia Research 83, 155-171 (2006).

[17] Hammers, A. et al. Three-dimensional maximum probability atlas of the human brain, with particular reference to the temporal lobe. Human Brain Mapping 19, 224-247 (2003).

[18] Thomas Yeo, B. T. et al. The organization of the human cerebral cortex estimated by intrinsic functional connectivity. Journal of Neurophysiology 106, 1125-1165 (2011).

[19] Schaefer, A. et al. Local-global parcellation of the human cerebral cortex from intrinsic functional connectivity mri. Cerebral Cortex 28, 3095-3114 (2018).

[20] Glasser, M. F. et al. A multi-modal parcellation of human cerebral cortex. Nature 536, 171 178 (2016).

21] Coalson, T. S., Van Essen, D. C. \& Glasser, M. F. The impact of traditional neuroimaging methods on the spatial localization of cortical areas. Proceedings of the National Academy of Sciences 115, E6356-E6365 (2018).

[22] Wu, Z., Xu, D., Potter, T., Zhang, Y. \& The, A. D. N. I. Effects of brain parcellation on the characterization of topological deterioration in alzheimer's disease. Frontiers in Aging Neuroscience 11, 113 (2019).

[23] Proix, T., Bartolomei, F., Guye, M. \& Jirsa, V. K. Individual brain structure and modelling predict seizure propagation. Brain : a journal of neurology 140, 641-654 (2017).

[24] Wirsich, J. et al. Whole-brain analytic measures of network communication reveal increased structure-function correlation in right temporal lobe epilepsy. Neurolmage. Clinical 11, 707718 (2016).

[25] Willner, P. The validity of animal models of depression. Psychopharmacology 83, 1-16 (1984).

[26] Bassett, D. S., Zurn, P. \& Gold, J. I. On the nature and use of models in network neuroscience. Nature Reviews Neuroscience 19, 566-578 (2018).

[27] Association, A. P. Technical recommendations for psychological tests and diagnostic techniques. Psychological bulletin 51, 1-38 (1954).

[28] Salehi, M. et al. There is no single functional atlas even for a single individual: Functional parcel definitions change with task. Neurolmage 208, 116366 (2020).

[29] Zalesky, A. et al. Whole-brain anatomical networks: does the choice of nodes matter? Neuroimage 50, 970-983 (2010).

[30] Cocchi, L. et al. Disruption of structure-function coupling in the schizophrenia connectome. Neurolmage. Clinical 4, 779-787 (2014)

[31] Sathian, K. \& Crosson, B. Structure-function correlations in stroke. Neuron 85, 887-889 (2015).

[32] Gorgolewski, K. J. et al. Neurovault.org: a web-based repository for collecting and sharing unthresholded statistical maps of the human brain. Frontiers in neuroinformatics 9, 8 (2015).

[33] Lawrence, R. M. et al. Standardizing human brain parcellations. Scientific data 8, 78 (2021).

[34] Alexander, B. et al. A new neonatal cortical and subcortical brain atlas: the melbourne children's regional infant brain (m-crib) atlas. Neurolmage 147, 841-851 (2017).

[35] Brennan, B. P. et al. Use of an individual-level approach to identify cortical connectivity biomarkers in obsessive-compulsive disorder. Biological psychiatry. Cognitive neuroscience and neuroimaging 4, 27-38 (2019).

[36] Cabezas, M., Oliver, A., Lladó, X., Freixenet, J. \& Cuadra, M. B. A review of atlas-based segmentation for magnetic resonance brain images. Computer methods and programs in biomedicine 104, e158-77 (2011).

[37] Caspers, S., Eickhoff, S. B., Zilles, K. \& Amunts, K. Microstructural grey matter parcellation and its relevance for connectome analyses. Neurolmage 80, 18-26 (2013).

[38] Diedrichsen, J., Balsters, J. H., Flavell, J., Cussans, E. \& Ramnani, N. A probabilistic mr atlas of the human cerebellum. Neurolmage 46, 39-46 (2009).

[39] Belzung, C. \& Lemoine, M. Criteria of validity for animal models of psychiatric disorders: focus on anxiety disorders and depression. Biology of mood \& anxiety disorders 1, 9 (2011).
[40] Association, A. E. R., Association, A. P., Education, N. C. o. M. i., (U.S., J. C. o. S. f. E. \& Testing, P. Standards for Educational and Psychological Testing (American Educational Association, 2014).

[41] Sporns, O., Tononi, G. \& Kötter, R. The human connectome: A structural description of the human brain. PLOS computational biology 1, e42 (2005)

[42] Fornito, A., Zalesky, A. \& Bullmore, E. Fundamentals of Brain Network Analysis (Academic Press, 2016).

[43] Sporns, O. The human connectome: a complex network. Annals of the New York Academy of Sciences 1224, 109-125 (2011).

[44] Greene, P., Li, A., González-Martínez, J. \& Sarma, S. V. Classification of stereo-eeg contacts in white matter vs. gray matter using recorded activity. Frontiers in Neurology 11 (2021).

[45] Mercier, M. R. et al. Evaluation of cortical local field potential diffusion in stereotactic electroencephalography recordings: A glimpse on white matter signal. Neurolmage 147, 219-232 (2017).

[46] Young, J. J. et al. Quantitative signal characteristics of electrocorticography and stereoelectroencephalography: The effect of contact depth. Journal of clinical neurophysiology : official publication of the American Electroencephalographic Society 36, 195-203 (2019).

[47] Revell, A. Y. et al. White matter signals reflect information transmission between brain regions during seizures. bioRxiv (2021)

[48] Henderson, M. X. et al. Spread of $\alpha$-synuclein pathology through the brain connectome is modulated by selective vulnerability and predicted by network analysis. Nat Neurosci $\mathbf{2 2}$ 1248-1257 (2019).

[49] Wang, H. et al. Vep atlas: An anatomic and functional human brain atlas dedicated to epilepsy patients. J Neurosci Methods 348, 108983 (2021).

[50] Doucet, G. et al. Atlas55+: Brain functional atlas of resting-state networks for late adulthood. Cereb Cortex 31, 1719-1731 (2021).

[51] Muñoz-Castañeda, R. et al. Cellular anatomy of the mouse primary motor cortex. Nature 598, 159-166 (2021).

[52] Joglekar, A. et al. A spatially resolved brain region- and cell type-specific isoform atlas of the postnatal mouse brain. Nat Commun 12, 463 (2021).

[53] Callaway, E. M. et al. A multimodal cell census and atlas of the mammalian primary motor cortex. Nature 598, 86-102 (2021).

[54] Amunts, K., Mohlberg, H., Bludau, S. \& Zilles, K. Julich-brain: A 3d probabilistic atlas of the human brain's cytoarchitecture. Science (New York, N. Y.) 369, 988-992 (2020).

[55] Wang, Q. et al. The allen mouse brain common coordinate framework: A $3 d$ reference atlas. Cell 181, 936-953.e20 (2020).

[56] Mišić, B. et al. Cooperative and competitive spreading dynamics on the human connectome. Neuron 86, 1518-1529 (2015).

[57] Betzel, R. F. et al. Structural, geometric and genetic factors predict interregional brain connectivity patterns probed by electrocorticography. Nature Biomedical Engineering (2019).

[58] Smith, R., Tournier, J., Calamante, F. \& Connelly, A. Sift2: Enabling dense quantitative assessment of brain white matter connectivity using streamlines tractography. Neuroimage 119, 338-351 (2015).

[59] Bijsterbosch, J., Smith, S. M. \& Beckmann, C. F. An Introduction to Resting State FMRI Functional Connectivity (Oxford University Press, 2017).

[60] A multimodal platform for cloud-based collaborative research (IEEE, 2013).

[61] Kini, L. G., Davis, K. A. \& Wagenaar, J. B. Data integration: Combined imaging and electrophysiology data in the cloud. Neurolmage 124, 1175-1181 (2016).

[62] Cieslak, M. et al. Qsiprep: an integrative platform for preprocessing and reconstructing diffusion mri data. Nat Methods 18, 775-778 (2021).

[63] Fang-Cheng, Y., Wedeen, V. J. \& Tseng, W.-Y. I. Generalized q-sampling imaging. IEEE Transactions on Medical Imaging 29, 1626-1635 (2010).

[64] Otsu, N. A threshold selection method from gray-level histograms. IEEE Transactions on Systems, Man, and Cybernetics 9, 62-66 (1979).

[65] Bonilha, L., Gleichgerrcht, E., Nesland, T., Rorden, C. \& Fridriksson, J. Gray matter axona connectivity maps. Frontiers in Psychiatry 6 (2015).

[66] Park, B., Eo, J. \& Park, H.-J. Structural brain connectivity constrains within-a-day variability of direct functional connectivity. Frontiers in Human Neuroscience 11, 408 (2017).

[67] Taylor, P. N. et al. The impact of epilepsy surgery on the structural connectome and its relation to outcome. Neurolmage. Clinical 18, 202-214 (2018).

[68] Fonov, V. et al. Unbiased average age-appropriate atlases for pediatric studies. Neurolmage 54, 313-327 (2011).

[69] Maslov, S. Specificity and stability in topology of protein networks. Science 296, 910-913 (2002).

[70] Litt, B. et al. Epileptic seizures may begin hours in advance of clinical onset. Neuron $\mathbf{3 0}$, 51-64 (2001).

[71] Azarion, A. A. et al. An open-source automated platform for three-dimensional visualization of subdural electrodes using ct-mri coregistration. Epilepsia 55, 2028-2037 (2014).

[72] Ludwig, K. A. et al. Using a common average reference to improve cortical neuron recordings from microelectrode arrays. Journal of neurophysiology 101, 1679-1689 (2009).

73] Kramer, M. A. et al. Coalescence and fragmentation of cortical networks during focal seizures. The Journal of neuroscience : the official journal of the Society for Neuroscience 30, 10076 10085 (2010).

[74] Khambhati, A. N. et al. Dynamic network drivers of seizure generation, propagation and termination in human neocortical epilepsy. PLoS computational biology 11, e1004608 (2015).

[75] Khambhati, A. N., Davis, K. A., Lucas, T. H., Litt, B. \& Bassett, D. S. Virtual cortical resection reveals push-pull network control preceding seizure evolution. Neuron 91, 1170-1182 (2016)

[76] Khambhati, A. N. et al. Recurring functional interactions predict network architecture of interictal and ictal states in neocortical epilepsy. eNeuro 4, ENEURO.0091-16.2017 (2017).

[77] Newson, J. J. \& Thiagarajan, T. C. Eeg frequency bands in psychiatric disorders: A review of resting state studies. Frontiers in Human Neuroscience 12, 521 (2019). 
bioRxiv preprint doi: https://doi.org/10.1101/2021.06.11.448063; this version posted October 7, 2021. The copyright holder for this preprint (which was not certified by peer review) is the author/funder, who has granted bioRxiv a license to display the preprint in perpetuity. It is made available under aCC-BY-NC 4.0 International license.

\section{Materials and Methods}

Human Dataset. MRI data was collected from forty-one individuals, including thirteen healthy controls and twenty-eight drug-resistant epilepsy patients at the Hospital of the University of Pennsylvania. Twenty-four patients underwent stereoelectroencephalography (SEEG) implantation and four underwent electrocorticography (ECoG) implantation. Ten of the SEEG patients had clinically annotated seizures and were used for SFC analyses. Inclusion criteria consisted of all individuals who agreed to participate in our research scanning protocol, and (if they had implantations) allowed their de-identified intracranial EEG (iEEG) data to be publicly available for research purposes on the International Epilepsy Electrophysiology Portal (https://www.ieeg.org) ${ }^{60,61}$. Seizure evaluation was determined via comprehensive clinical assessment, which included multimodal imaging, scalp and intracranial video-EEG monitoring, and neuropsychological testing. This study was approved by the Institutional Review Board of the University of Pennsylvania, and all subjects provided written informed consent prior to participating. See Table S2 for subject demographics.

Structure. Methods and pipelines for structural connectivity generation and analysis are described in the following sections. Specific GitHub files and code are included where applicable.

Imaging Protocol. Prior to electrode implantation, MRI data were collected on a $3 \mathrm{~T}$ Siemens Magnetom Trio scanner using a 32channel phased-array head coil. High-resolution anatomical images were acquired using a magnetization prepared rapid gradient echo (MPRAGE) T1-weighted sequence (repetition time $=1810 \mathrm{~ms}$, echo time $=3.51 \mathrm{~m}$, flip angle $=9$, field of view $=240 \mathrm{~mm}$, resolution $=$ 0.94x0.94x1.0 mm3). High Angular Resolution Diffusion Imaging HARDI) was acquired with a single-shot EPI multi-shell diffusionweighted imaging (DWI) sequence (116 diffusion sampling directions b-values of $0,300,700$, and $2000 \mathrm{~s} / \mathrm{mm} 2$, resolution $=2.5 \times 2.5 \times 2.5$ $\mathrm{mm} 3$, field of view $=240 \mathrm{~mm}$ ). Following electrode implantation, spiral CT images (Siemens) were obtained clinically for the purposes of electrode localization. Both bone and tissue windows were obtained $(120 \mathrm{kV}, 300 \mathrm{~mA}$, axial slice thickness $=1.0 \mathrm{~mm})$

Diffusion Weighted Imaging (DWI) Preprocessing. HARDI images were subject to the preprocessing pipeline, QSIPrep, to ensure reproducibility and implementation of the best practices for processing of diffusion images ${ }^{62}$. Briefly, QSIPrep performs advanced reconstruction and tractography methods in curated workflows using tools from leading software packages, including FSL, ANTs, and DSI Studio with input data specified in the Brain Imaging Data Structure (BIDS) layout.

\section{Structural Network Generation. DSI-Studio (http://dsi-} studio.labsolver.org, version: December 2020) was used to reconstruct the orientation density functions within each voxel using generalized q-sample imaging with a diffusion sampling length ratio of $1.25^{63}$. Deterministic whole-brain fiber tracking was performed using an angular threshold of 35 degrees, step size of $1 \mathrm{~mm}$, and quantitative anisotropy threshold based on Otsu's threshold ${ }^{64}$. Tracks with length shorter than $10 \mathrm{~mm}$ or longer than $800 \mathrm{~mm}$ were discarded, and a total of 1,000,000 tracts were generated per brain. Deterministic tractography was chosen based upon prior work indicating that deterministic tractography generates fewer false positive connections than probabilistic approaches, and that network-based estimations are substantially less accurate when false positives are introduced into the network compared with false negatives ${ }^{29}$. To calculate structural connectivity, atlases listed in Table 1 were used. Structural networks were generated by computing the number of streamlines passing through each pair of structural regions in each specific atlas. Streamline counts were log-transformed and normalized to the maximum streamline count, as is common in prior studies ${ }^{24,65-67}$. GitHub: packages/imaging/tractography/tractography.py

Atlases. Atlas descriptions and sources used in this study are found in Table S1. All atlases were sourced in MNI space and if not already, resliced to dimensions $182 \times 218 \times 182$. Atlases were linear then non-linear registered to T1w subject space using the ICBM 2009c Nonlinear Asymmetric template ${ }^{68}$ and FSL flirt and fnirt. In addition to published standard atlases demarcating neuroanatomical and functional boundaries, we used whole-brain random atlases. A limitation of most standard atlases is that they may not have anatomical definitions for all regions of the brain, and therefore, implanted electrodes may not be assigned properly to a region. Whole-brain random atlases, in contrast, provide coverage to all implanted electrodes. They also allow for the ability to change some morphological properties (i.e. parcellation size), while keeping other morphologies the same (i.e. parcellation shape; Fig. 2d). A limitation of random atlases is that regions may not represent true anatomical or functional boundaries. With the limitations for each approach in mind, analyses were conducted for both standard and random atlases. Random atlases were built in the ICBM template space and covered all voxels, excluding those labeled as CSF or outside the template. To fill these points, a pseudo grassfire algorithm was applied ${ }^{29}$. Briefly, $\mathrm{N}$ points representing the number of regions of interests (ROIs) of the atlas were randomly chosen as seed points. These seed points were iteratively expanded in all six Cartesian directions until all points were covered by one of the initial $\mathrm{N}$ seeds. After each iterative step, the smallest volume region expanded first. Random atlases created were of $\mathrm{N}$ equal to $10,30,50,75,100,200,300$, $400,500,750,1000,2000,5000$, and 10000 ROIs. Five permutations for each $\mathrm{N}$ were created. GitHub code to generate random atlases: packages/imaging/randomAtlas/randomAtlasGeneration.py

Atlas Morphology: Volume and Sphericity. Atlas morphological measurements included regions of interest (ROI) size and shape, and were measured with volume and sphericity calculations, respectively (Fig. 2). Region volume was calculated as the number of voxels in an ROI and $\log 10$ transformed. Region sphericity was calculated as the ratio of the surface area of a sphere with an equal volume of the ROI to the actual surface area of the atlas ROI. Under this definition, sphericity is bounded from 0 to 1 where 1 is a perfect sphere. For reference, a perfect cube and a hemi-sphere have a sphericity of 0.8 and 0.7 respectively. GitHub: packages/imaging/regionMorphology/regionMorphology.py

Structural Network Measures. We characterized the structural network topology of 52 atlases (Fig. 3 and Fig. S3). To quantify network topology, we examined density, mean degree, mean clustering coefficient, characteristic path length, and small worldness. Connectivity matrices were first binarized, using a threshold of 0 , and a distance matrix was computed. The same binarization process and threshold was used across all atlases. The distance of any nodes that were disconnected from the main graph was set to the maximum distance between any pair of nodes in the main graph. Density, mean degree, clustering coefficient, and characteristic path length were then calculated on the binary, undirected graphs. Small worldness was calculated as the $\sigma$-ratio where $\sigma=\gamma / \lambda$ and is the ratio of the average, normalized clustering coefficient, $\mathrm{C}$, to the normalized characteristic path length, I. $\gamma=\mathrm{CG} / \mathrm{CR}$ and $\lambda=\mathrm{lG} / \mathrm{lR}$ where $\mathrm{G}$ is the graph of interest and $\mathrm{R}$ represents a 'random' graph that is equivalent to $\mathrm{G}$. To approximate the equivalent random graph $\mathrm{R}$ due to intractable computational costs ${ }^{69}$, a well-known analytical equivalent $\mathrm{CR}=\mathrm{d} / \mathrm{N}$ and $\mathrm{IR}=\log \mathrm{N} / \log \mathrm{d}$ were used, where $\mathrm{d}$ denotes average nodal degree. All network measures were calculated using the Brain Connectivity Toolbox for Python. GitHub: papers/brainAtlas/Script_05_structure_02_network_measures.py

Function. Methods and pipelines for functional connectivity generation and analysis are described in the following sections. Specific GitHub files and code are included where applicable.

Intracranial EEG Acquisition. Stereotactic Depth Electrodes were implanted in patients based on clinical necessity. Continuous SEEG signals were obtained for the duration of each patient's stay in the epilepsy monitoring unit. Intracranial data was recorded at either 512 or $1024 \mathrm{~Hz}$ for each patient. Seizure onset times were defined by the unequivocal onset ${ }^{70}$. All annotations were verified and consistent with detailed clinical documentation. If a patient had more than one seizure annotated, the first seizure longer than 30 seconds without artifacts was used. iEEG times used in the study are 
sourced in the Box folder (below) and iEEG snippets are downloaded using GitHub script in packages/eeg/ieegOrg/downloadiEEGorg.py

Electrode Localization. In-house software ${ }^{71}$ was used to assist in localizing electrodes after registration of pre-implant and post-implant neuroimaging data. All electrode coordinates and labels were saved and matched with the electrode names on IEEG.org. All electrode localizations were verified by a board-certified neuroradiologist (J.S.). Electrode coordinates in patient $\mathrm{T} 1 \mathrm{w}$ space were assigned to an atlas ROI also registered in patient T1w space. To assign electrodes to an atlas ROI, we followed the same protocol in Shah et al., $2019^{13}$ Electrode coordinates in patient $\mathrm{T} 1 \mathrm{w}$ space were transformed to MNI space, where atlases were also defined. Atlas ROI assignment was performed by rounding electrode coordinates $(\mathrm{x}, \mathrm{y}, \mathrm{z})$ to the nearest voxel and indexing the given atlas at that voxel. Electrodes that fell outside the atlas of interest were excluded from subsequent analysis. GitHub: packages/atlasLocalization/atlasLocalization.py

Functional Connectivity Network Generation. Functional connectivity networks were generated from four periods: interictal, preictal, ictal, and postictal. (1) The interictal period consisted of the time approximately 6 hours before the ictal period. (2) The preictal period consisted of the time immediately before the ictal period. (3) The ictal period consisted of the time between the seizure unequivocal onset and seizure termination. (4) The postictal period consisted of the time immediately after the ictal period. Interictal, preictal, and postictal periods were 180 seconds in duration. Following removal of artifact-ridden electrodes, SEEG signals inside either GM or WM for each period were common-average referenced to reduce potential sources of correlated noise ${ }^{72}$. Next, each period was divided into $2 \mathrm{~s}$ time windows with $1 \mathrm{~s}$ overlap ${ }^{73-76}$. To generate a functional network representing broadband functional interactions between SEEG signals (Fig. 4b), we carried out a method described in detail previously ${ }^{13,75}$. Namely, signals were notch-filtered at 60 $\mathrm{Hz}$ to remove power line noise, low-pass and high-pass filtered at 127 $\mathrm{Hz}$ and $1 \mathrm{~Hz}$ to account for noise and drift, and pre-whitened using a first-order autoregressive model to account for slow dynamics. Functional networks were then generated by applying a normalized cross correlation function $\rho$ between the signals of each pair of electrodes within each time window, using the formula:

$$
\rho_{x y}=\max _{\tau}\left[\frac{1}{T} \sum_{t=1}^{T} \frac{\left[x_{k}(t)-\bar{x}_{k}\right] *\left[y_{k}(t+\tau)-\bar{y}_{k}\right]}{\sigma_{x_{k}} \sigma_{y_{k}}}\right]
$$

where $\mathrm{x}$ and $\mathrm{y}$ are signals from two electrodes, $\mathrm{k}$ is the $2 \mathrm{~s}$ time window, $\mathrm{t}$ is one of the $\mathrm{T}$ samples during the time window, and $\tau$ is the time lag between signals, with a maximum lag of $0.5 \mathrm{~s}$. Here, $\sigma$ represents the standard deviation of the signal. Functional connectivity measurements were also calculated for coherence and zero time-lag Pearson and Spearman rank correlations with associated p-values. They are included in the freely available open source dataset but were not used in hypothesis testing in the study. Also freely available are the functional connectivity measurements in defined frequency bands reviewed in Newson and Thiagarajan $2019^{77}$. Networks are represented as full-weighted connectivity matrices. GitHub Code: GitHub: code/tools/echobase.py

Structure-Function Correlation. To quantify the relationship between structure and function in the epileptic brain, we computed the Spearman rank correlation coefficient between the edges of the structural connectivity network and the edges of the functional connectivity networks (Fig. 4c). To avoid redundancy given the symmetric nature of the matrices, only the upper triangle was analyzed. In brief, the structural connectivity network, representing normalized streamline counts between each atlas ROI, was first down sampled to only include ROIs that contained at least one SEEG contact. This gave one static representation of structural connectivity. In the case where multiple electrodes fell in the same atlas ROI, a random electrode was selected to represent the functional activity of that neuroanatomically defined region. Next, for every time-window of the functional network, the functional network edges were correlated with the down sampled, static structural network edges. This resulted in a structure-function correlation time series. Note that atlases with very small ROI volumes included more electrodes for SFC calculation. Electrodes that did not localize to an atlas were excluded from analysis. To average the SFC for all patients and each atlas (Fig. 5), SFC time-series was resampled to 100 seconds for each period and each sample was averaged together. GitHub code: packages/eeg/echobase/echobase.py

rsSFC and $\triangle$ SFC. Resting-state SFC (rsSFC) was defined as the SFC during the interictal period, approximately 6 hours before the ictal period. The mean SFC of that period was computed. $\triangle \mathrm{SFC}$ was defined as the change in the mean SFC from the preictal to the ictal period (Fig. 5 top left panel). rsSFC and $\triangle \mathrm{SFC}$ was calculated for each atlas (Fig. 6).

Statistics. Preictal and ictal SFC for each atlas were compared using effect sizes across the 52 atlases shown in Fig. 6d. Cohen's d and the difference between preictal and ictal SFC was calculated.

Data availability and Reproducibility. All code files used in this manuscript are available at https://github.com/andyrevell/revellLab. All de-identified raw and processed data (except for patient MRI imaging) are available for download on Box. Link provided on GitHub. Raw imaging data is available upon reasonable request from Principal Investigator K.A.D.; tractography files generated from the imaging data are readily available on Box. iEEG snippets used specifically in this manuscript are contained within the Box data folder, while full iEEG recordings are publicly available at https://www.ieeg.org. The Python environment for the exact packages and versions used in this study in contained in the environment directory within the GitHub. The QSIPrep docker container was used for DWI preprocessing.

\section{Acknowledgements}

We thank Adam Gibson, Carolyn Wilkinson, Jacqueline Boccanfuso, Magda Wernovsky, Ryan Archer, Kelly Oechsel, members of Andrew's Thesis Committee, and all other members and staff of the Center for Neuroengineering and Therapeutics for their continued help and support in this work.

\section{Funding}

This work was supported by National Institutes of Health grants 5T32-NS-091006-07, 1R01NS116504, 1R01NS099348, 1R01NS085211, and 1R01MH112847. We also acknowledge support by the Thornton Foundation, the Mirowski Family Foundation, the ISI Foundation, the John D. and Catherine T. MacArthur Foundation, the Sloan Foundation, the Pennsylvania Tobacco Fund, and the Paul Allen Foundation.

\section{Competing Interests}

The authors declare no competing interests. 


\section{Supplementary Material}

Please see supplemental figures and tables contained below.

\section{- Figures}

- Fig. S1: Atlas, Template, and Coordinate (Stereotactic) Space

- Fig. S2: Atlas Morphology: Sizes and Shapes (All atlases)

- Fig. S3: Network measures for remaining atlases

- Fig. S4: Network measures for controls and patients separated

- Fig. S5: Network measures for different thresholds

- Fig. S6: Coverage of electrode contacts

- Fig. S7: "Brain Atlas" Search in PubMed

\section{- Tables}

- Table. S1: Atlas Sources and References (3 pages).

- Table. S2: Patient and Control Demographics

- Other materials

- Glossary

Glossary.

1. Atlas abbreviations and definitions. For further details, see Table. S1.

(a) AAL. Automated anatomical labeling atlas.

(b) AAL1, AAL2, AAL3. AAL atlas versions 1, 2, and 3, respectively.

(c) AAL-JHU. The AAL atlas and the JHU labels atlas combined. For overlapping regions, the JHU atlas takes precedence.

(d) AAL600. AAL atlas with 600 parcels.

(e) AICHA. Atlas of Intrinsic Connectivity of Homotopic Areas.

(f) BNA. Brainnetome atlas.

(g) Craddock 200-400. Craddock atlases with a specified number of parcels (e.g. Craddock 200 will have 200 parcels). There are two atlas sizes publicly available the Craddock 200 and Craddock 400 atlases.

(h) DKT31 OASIS. The DKT atlas from the OASIS dataset. See Table. S1 sources for more details.

(i) HO. Harvard-Oxford atlas.

(j) HO cortical-only. HO atlas with only cortical regions. The symmetrical regions (the same region name on the contralateral hemisphere) are labeled with different identifications. Thus, this atlas has non-symmetrical labels (e.g. both temporal pole regions are labeled with a different identification number). Left and right structures were re-labeled with different identification numbers using the sagittal mid-line (in MNI space, $\mathrm{x}$ coordinate at zero) as a separator.

(k) HO cort-only. Same as the HO cortical-only atlas.

(l) HO sym. cortical only. HO atlas with only cortical regions. The symmetrical regions (the same region name on the contralateral hemisphere) are labeled with the same identification. Thus, this atlas is has symmetrical labels (e.g. both temporal pole regions are labeled with the same identification number). The default atlases given by FSL are symmetrical atlases. (m) HO subcortical-only. HO atlas with only subcortical regions.

(n) HO subcort-only. Same as the HO subcortical-only atlas.

(o) HO combined. HO atlas with both cortical and subcortical regions. This atlas has non-symmetrical labeling (e.g. both temporal pole regions are labeled with a different identification number).

(p) HO cortical + subcortical. Same as the HO combined atlas.

(q) JHU. The Johns Hopkins University atlases. There are two white matter atlases: thee JHU labels and JHU tracts atlases.

(r) MMP. Multi-modal parcellation atlas. Sometimes referred to as the "Glasser Atlas" after the first author of the original publication.

(s) Random atlas 10-10,000. Atlases created with random parcels with a specified number of parcels (e.g. Random atlas 1,000 will have 1,000 parcels). These atlases were built in the ICBM 2009c Nonlinear Asymmetric template. Thus, these atlases are whole-brain atlases (includes cortical gray matter, subcortical gray matter, and white matter). See the 'Atlases' Methods section for more details.

(t) Schaefer 100-1,000. The Schaefer atlases with a specified number of parcels (e.g. Schaefer 100 will have 100 parcels). There are ten atlases of 100, 200, 300, 400, 500, $600,700,800,900$, and 1,000 parcels.

(u) Yeo liberal. The Yeo atlases where the boundaries of each parcel is extended slightly into the white matter, past the cortical boundary.

(v) Yeo conservative. The Yeo atlases where the boundaries of each parcel is extended slightly into the white matter, past the cortical boundary.

2. $\triangle$ SFC. The change in SFC between ictal and preictal stats $\left(S F C_{i c t a l}-S F C_{\text {preictal }}\right)$. This indicates whether or not the change in functional connectivity is congruent with the underlying structural connectivity.

3. Contact. A single sensor on an electrode that records LFP. Not to be confused with an electrode. See Fig. S6, bottom.

4. ECoG: Electrocorticography.

5. Electrode. Not to be confused with contact. See Fig. S6, bottom.

6. Functional connectivity (FC). The statistical relationship between two signals (two contacts in this study).

7. grayordinate. Atlas that includes gray matter structures, including cortical and subcortical gray matter regions.

8. ROI. Region of interest

9. ROI, parcel, parcellation, region. These terms may be used interchangeably in the literature. They refer to discrete areas of a brain. These regions are labeled with a categorical identification (rather than a continuous variable seen in templates - see Fig. S1), and all voxels or surface vertices with the same identification are part of thee same region.

10. SEEG: Stereoelectroeenccephalography.

11. Structural connectivity (SC). The physical relationship between two brain regions. We use streamline counts in this manuscript from High Angular Resolution Diffusion Imaging.

12. T1w. T1-weighted MRI image. 
bioRxiv preprint doi: https://doi.org/10.1101/2021.06.11.448063; this version posted October 7, 2021. The copyright holder for this preprint (which was not certified by peer review) is the author/funder, who has granted bioRxiv a license to display the preprint in perpetuity. It is made available under aCC-BY-NC 4.0 International license.

a

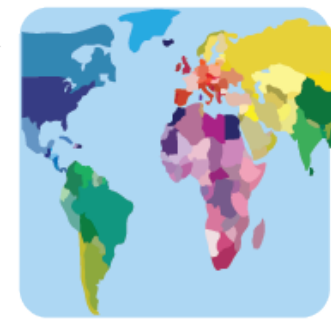

Atlas

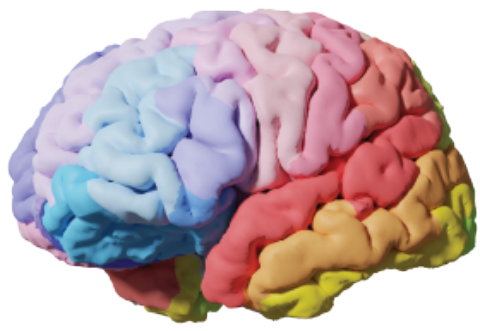

\section{Clarifying Terminologies}
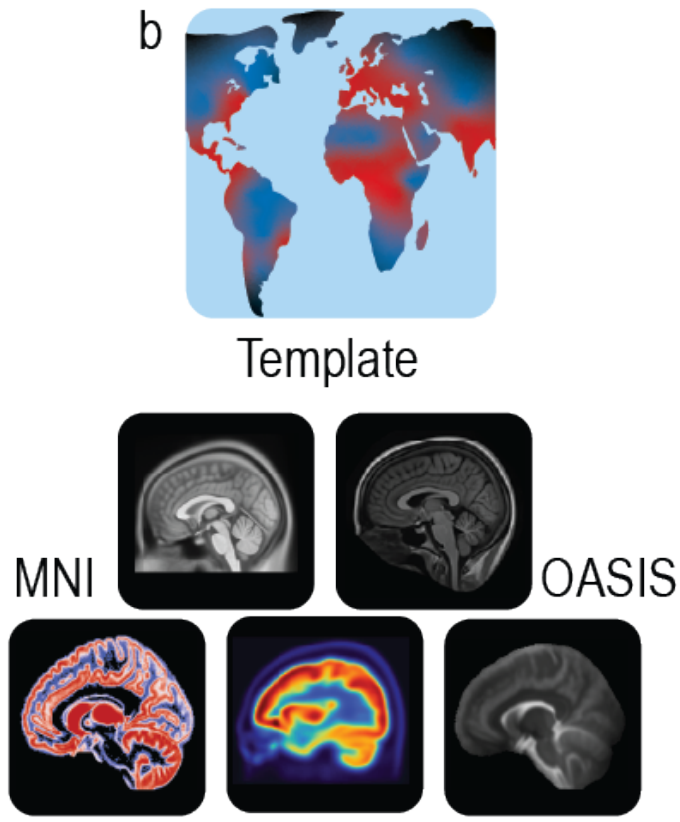

PET

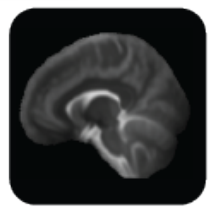

DTI
C

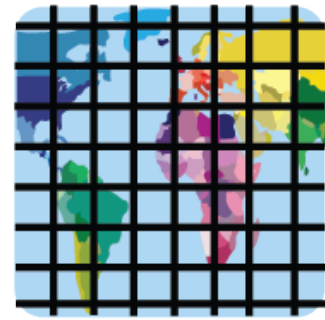

Coordinate System

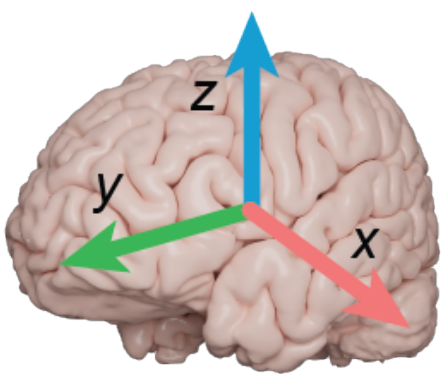

Fig. S1. Atlas, Template, and Coordinate (Stereotactic) Space. | These three terms are commonly confused in the neuroscience literature because they all relate to the "map" of the brain. "Atlas" and "template" are sometimes used interchangeably $^{3}$, however, they are distinct. Here, we define them more formally. a, A brain atlas refers to a neurological map that defines brain region labels. We use this definition throughout the main text. $\mathbf{b}$, An atlas is distinct from a brain template, which refers to a brain pattern. Similar in common usage, a template is a mold, gauge, or starting point representation of the brain. Usually it is composed of multiple individuals' brain representing an average of a population. Many templates exist and are reviewed in various publications ${ }^{2,9}$, The templates illustrated here are the MNI152 Nonlinear asymmetric $2009 \mathrm{c}$ T1w template (http://www.bic.mni.mcgill.ca), the OASIS brain template https://www.oasis-brains.org/ created and used by ANTs (http://stnava.github.io/ANTs/ with templates linked here), a gray matter probability map, a PET template, and a b0 DTI template. c, The coordinate system, or the stereotactic space, of the brain describes the physical positioning of the brain, similar to the geographical coordinate system of longitude and latitude of the Earth. Historically, a common stereotactic space was the Talairach space, and more recently, the MNI spaces. The analogy between the geographical terms of the Earth and the geographical terms of the brain is not exact. The analogy falls apart in that while there in one world, there are many brains. There is variability across populations and a spectrum of differences between species, therefore, it is challenging to represent one brain for use in every scientific study appropriately. MNI, Montreal Neurological Institute; OASIS, Open Access Series of Imaging Studies; GM, Gray Matter probability map; PET, Positron Emission Tomography; DTI, Diffusion Tensor Imaging. 
bioRxiv preprint doi: https://doi.org/10.1101/2021.06.11.448063; this version posted October 7, 2021. The copyright holder for this preprint (which was not certified by peer review) is the author/funder, who has granted bioRxiv a license to display the preprint in perpetuity. It is made available under aCC-BY-NC 4.0 International license.

\begin{tabular}{|c|c|c|c|}
\hline Atlas & Source & Note & Reference(s) \\
\hline \multirow[t]{7}{*}{ AAL } & 1 & $\begin{array}{l}\text { AAL1. The successor to the Talairach atlas. The goal was to } \\
\text { reduce confusion in relating stereotaxic space (a set of brain } \\
\text { coordinates) and anatomical labels. It is based on a single indi- } \\
\text { vidual (the Collin-27 template) and it is not a probabilistic map. } \\
\text { The Collin-27 template was intended for segmentation, and not } \\
\text { stereotaxy; it did not capture anatomical variability. However, } \\
\text { the high resolution in } 1998 \text { proved attractive to research groups. }\end{array}$ & $\begin{array}{l}\text { (1) Tzourio-Mazoyer, N. et al. Automated Anatomical Labeling of Activations in SPM } \\
\text { Using a Macroscopic Anatomical Parcellation of the MNI MRI Single-Subject Brain. } \\
\text { Neurolmage 15, 273-289 (2002). } \\
\text { (2) Collin-27 template: Holmes, C. J. et al. Enhancement of MR Images Using } \\
\text { Registration for Signal Averaging: Journal of Computer Assisted Tomography 22, } \\
\text { 324-333 (1998). } \\
\text { (3) Website about Collin-27: https://www.bic.mni.mcgill.ca/ServicesAtlases/Colin27 }\end{array}$ \\
\hline & 2 & $\begin{array}{l}\text { AAL2: new parcellation of orbitofrontal cortex. AAL1 orbitofron- } \\
\text { tal cortex was parcellated according to a French publication } \\
\text { by Jules Déjerine in } 1895 \text {. Chiavaras and Petrides ( } 2000) \\
\text { proposed another parcellation of the orbital surface allowing } \\
\text { for the comparison of human frontal lobe anatomy with that of } \\
\text { macaques. }\end{array}$ & $\begin{array}{l}\text { (1) Rolls, E. T., Joliot, M. \& Tzourio-Mazoyer, N. Implementation of a new } \\
\text { parcellation of the orbitofrontal cortex in the automated anatomical labeling atlas. } \\
\text { Neurolmage 122, 1-5 (2015). } \\
\text { (2) Chiavaras, M. M. \& Petrides, M. Orbitofrontal sulci of the human and macaque } \\
\text { monkey brain. The Journal of Comparative Neurology 422, 35-54 } \\
\text { (3) Dejerine, J. Anatomie des centres nerveux. (Rueff Paris, 1895). }\end{array}$ \\
\hline & 3 & $\begin{array}{l}\text { AAL3: new parcellations - anterior cingulate, thalamus, nucleus } \\
\text { accumbens, substantia nigra, ventral tegmental area, red } \\
\text { nucleus, locus coeruleus, and raphe nuclei. } 2019 \text {. } \\
\text { AAL3v1: changes of thalamus in line with FreeSurfer } 7.2020 .\end{array}$ & $\begin{array}{l}\text { Rolls, E. T., Huang, C.-C., Lin, C.-P., Feng, J. \& Joliot, M. Automated anatomical } \\
\text { labelling atlas 3. Neurolmage 206, } 116189 \text { (2020). }\end{array}$ \\
\hline & 4 & $\begin{array}{l}\text { Website for download - group that made AAL toolbox and user } \\
\text { guides. }\end{array}$ & https://www.gin.cnrs.fr/en/tools/aal/ \\
\hline & 5 & $\begin{array}{l}\text { SPM - software compatible with AAL toolbox. Generally, } \\
\text { designed for the analysis of brain imaging data sequences. } \\
\text { Extensions include AAL toolbox. }\end{array}$ & $\begin{array}{l}\text { (1) Statistical parametric mapping: the analysis of functional brain images. (Elsevi- } \\
\text { er/Academic Press, 2007). } \\
\text { (2) Website: https://www.fil.ion.ucl.ac.uk/spm/ext/ }\end{array}$ \\
\hline & 6 & $\begin{array}{l}\text { AAL } 600 \text { - Subparcellations of the AAL atlas into } 600 \text { subre- } \\
\text { gions. Upsampling algorithm described. Part of larger frame- } \\
\text { work for evaluating the effect of parcellation scale. }\end{array}$ & $\begin{array}{l}\text { Bassett, D. S., Brown, J. A., Deshpande, V., Carlson, J. M. \& Grafton, S. T. Con- } \\
\text { served and variable architecture of human white matter connectivity. Neurolmage } \\
54,1262-1279 \text { (2011) }\end{array}$ \\
\hline & 7 & $\begin{array}{l}\text { Use cases of AAL } 600 \text {. Both Ashourvan et al. (2017) and } \\
\text { Hermundstad et al. (2014) use AAL } 600 \text { for generating both } \\
\text { structural and functional connectivity networks. }\end{array}$ & $\begin{array}{l}\text { (1) Ashourvan, A., Telesford, Q. K., Verstynen, T., Vettel, J. M. \& Bassett, D. S. } \\
\text { Multi-scale detection of hierarchical community architecture in structural and } \\
\text { functional brain networks. (2017) } \\
\text { (2) Hermundstad, A. M. et al. Structurally-Constrained Relationships between } \\
\text { Cognitive States in the Human Brain. PLoS Comput Biol 10, e1003591 (2014). }\end{array}$ \\
\hline $\mathrm{Al}$ & 8 & $\begin{array}{l}\text { AICHA tries to account for homotopy. the concept that each } \\
\text { region in one hemisphere has a homologue in the other. }\end{array}$ & $\begin{array}{l}\text { Joliot, M. et al. AICHA: An atlas of intrinsic connectivity of homotopic areas. Journal } \\
\text { of Neuroscience Methods 254, 46-59 (2015) }\end{array}$ \\
\hline \multirow[t]{2}{*}{ Brainn } & 9 & $\begin{array}{l}\text { Connectivity-based atlas. Further subdivision of structural } \\
\text { parcellations using the DK (Desikan-Killiany) protocol, with } \\
\text { adjustments. }\end{array}$ & $\begin{array}{l}\text { Fan, L. et al. The Human Brainnetome Atlas: A New Brain Atlas Based on Connec- } \\
\text { tional Architecture. Cerebral cortex (New York, N.Y. : 1991) 26, 3508-26 (2016). } \\
\text { Website: http://atlas.brainnetome.org }\end{array}$ \\
\hline & 10 & $\begin{array}{l}\text { DSI studio created by Fang-Cheng (Frank) Yeh. Many recon- } \\
\text { struction and tracking algorithms are published and incorporat- } \\
\text { ed into DSI Studio. See citations page on website. Many atlases } \\
\text { available, including Brainnetome. Can use custom atlas. }\end{array}$ & $\begin{array}{l}\text { (1) Website: http://dsi-studio.labsolver.org/ } \\
\text { (2) Example of reconstruction method: Fang-Cheng Yeh, Wedeen, V. J. \& } \\
\text { Tseng, W.-Y. I. Generalized q-Sampling Imaging. IEEE Trans. Med. Imaging 29, } \\
\text { 1626-1635 (2010). }\end{array}$ \\
\hline \multirow[t]{3}{*}{ Brodm } & 11 & $\begin{array}{l}\text { Perspective, description, and historical significance of Korbinian } \\
\text { Brodman's map. }\end{array}$ & $\begin{array}{l}\text { Zilles, K. \& Amunts, K. Centenary of Brodmann's map - conception and fate. Nat } \\
\text { Rev Neurosci 11, 139-145 ( } 2010\end{array}$ \\
\hline & 12 & $\begin{array}{l}\text { References to the original German and English translation } \\
\text { provided. }\end{array}$ & $\begin{array}{l}\text { (1) Original German: Vergleichende Lokalisationslehre der Grosshirnrinde in ihren } \\
\text { Prinzipien dargestellt auf Grund des Zellenbaues. (1909) } \\
\text { (2) English translation: Brodmann, K. \& Gary, L. J. Brodmann's localisation in the } \\
\text { cerebral cortex: the principles of comparative localisation in the cerebral cortex } \\
\text { based on cytoarchitectonics. (Springer, } 2006\end{array}$ \\
\hline & 13 & $\begin{array}{l}\text { The atlas is available through MRIcro, a legacy tool developed } \\
\text { by Chris Rorden (University of South Carolina). The atlas is } \\
\text { based on work from the Van Essen lab (Washington University } \\
\text { in St. Louis) with corresponding Talairach coordinates, and } \\
\text { transformed by Krish Singh (Cardiff University) to MNI space. }\end{array}$ & $\begin{array}{l}\text { (1) Chris Rorden legacy tools webpage: https://people.cas.sc.edu/rorden/ } \\
\text { (2) Updated webpage: https://crnl.readthedocs.io/ } \\
\text { (3) About Brodmann atlas: https://people.cas.sc.edu/rorden/mricro/lesion.html } \\
\text { (4) BALSA: https://balsa.wustl.edu/Wz8r }\end{array}$ \\
\hline $\mathrm{C}$ & 14 & $\begin{array}{l}\text { Introduction to the CerebrA and MNI-ICBM2009c average brain } \\
\text { template. }\end{array}$ & $\begin{array}{l}\text { Manera, A. L., Dadar, M., Fonov, V. \& Collins, D. L. CerebrA, registration and } \\
\text { manual label correction of Mindboggle-101 atlas for MNI-ICBM152 template. Sci } \\
\text { Data 7, 237 (2020). } \\
\text { Website: https://doi.gin.g-node.org/10.12751/g-node.be5e62 }\end{array}$ \\
\hline \multirow[t]{3}{*}{ Cradd } & 15 & Original publication about functional parcellations. & $\begin{array}{l}\text { Craddock, R. C., James, G. A., Holtzheimer, P. E., Hu, X. P. \& Mayberg, H. S. A } \\
\text { whole brain fMRI atlas generated via spatially constrained spectral clustering. Hum. } \\
\text { Brain Mapp. 33, 1914-1928 (2012). }\end{array}$ \\
\hline & 16 & Github with source code to make atlas with $\mathrm{N}$ clusters. & GitHub: http://ccraddock.github.io/cluster_roi/atlases.html \\
\hline & 17 & $\begin{array}{l}\text { Publicly available pre-made atlases at } \mathrm{N}=200 \text { and } \mathrm{N}=400 \text { from } \\
\text { ABIDE (Autism Brain Imaging Data Exchange), co-founded by } \\
\text { Cameron Craddock. } 4 \times 4 \times 4 \mathrm{~mm} \text { resolution. }\end{array}$ & ABIDE: http://preprocessed-connectomes-project.org/abide/Pipelines.html \\
\hline
\end{tabular}

Table S1. Atlas sources and references. | This table provides a short note and references to the source material of common atlases in the neuroscience literature. See also Table 1. 
bioRxiv preprint doi: https://doi.org/10.1101/2021.06.11.448063; this version posted October 7, 2021. The copyright holder for this preprint (which was not certified by peer review) is the author/funder, who has granted bioRxiv a license to display the preprint in perpetuity. It is made available under aCC-BY-NC 4.0 International license.

\begin{tabular}{|c|c|c|}
\hline Atlas & Source & Note \\
\hline \multirow[t]{3}{*}{ DKT } & 18 & $\begin{array}{l}\text { Original DK protocol and atlas. A protocol for an atlas is a set } \\
\text { of instructions for how the brain should be labeled. See AAL, } \\
\text { Hammersmith, Harvard-Oxford, and JHU atlases. }\end{array}$ \\
\hline & 19 & DKT protocol, Mindboggle-101 dataset, and atlas creation. \\
\hline & 20 & $\begin{array}{l}\text { Summary of Mindboggle project, history, atlas development, } \\
\text { applications, and current problems. }\end{array}$ \\
\hline
\end{tabular}

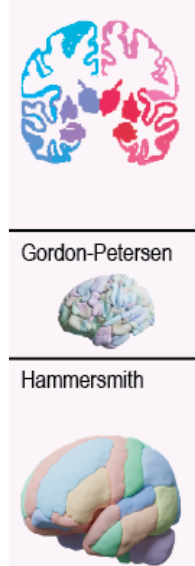

21 Websites for downloading data including the labeled brains and atlases.

22 Subcortical regions.

23 FreeSurfer.

24 Original article.

25 Resource to download atlas.

26 Original article (for regions 1-49), including their Hammersmith protocol (or "algorithm").

27 Updated regions (for regions 50-83).

28 Download atlas with 83 regions.

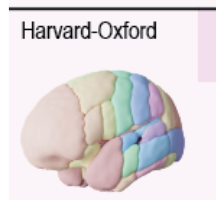

29
Atlas developed at the Center for Morphometric Analysis (CMA)
at Massachusetts General Hospital and distributed with FSL.

30 Individual segmentations were segmented by CMA using inhouse software. Probability maps were then created. Freesurfer link (right) has archived CMA's website and contains the Harvard-Oxford labeling protocols.

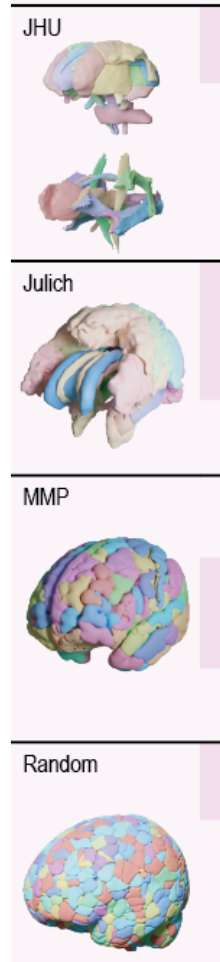

31 JHU labels: Protocol to reconstruct eleven white matter tracts and their segmentation into ROI labels. Included in FSL.

32 JHU Tracts: white matter parcellation atlas based on DTI probabilistic tractography of 11 major white matter tracts $\mathrm{d}$. Protoco defining manually identified ROls from which the tracts were formed are described in Wakana et al. (2005). Included in FSL.

33 Textbook with more information about these atlases.

34 Cytoarchitecture map. Successor to both the Brodmann and Eickhoff-Zilles atlases. The Eichoff-Zilles is an SPM toolbox (see note is source 5 about the AAL atlas) for probabilistic cytoarchitecture.

35 Website for the Julich Atlas and SPM toolbox

36 Original article on multi-modal approach

37 Information on surface vs volume based methodologies for localization of neuroanatomy.

38 Website to download data. Volumetric version also included in DSI-studio. Note the volume note above

39 Random atlas algorithm (pseudo-grassfire algorithm).

40 Use case of random atlas. Goni et al. (2014) study the structure-function relationship in the brain with tractography and fMRI. They used random cortical atlases of 1170 equally sized regions. Misic et al. (2015) used random cortical atlases of 1015 equally sized regions.
Reference(s)

Desikan, R. S. et al. An automated labeling system for subdividing the human cerebral cortex on MRI scans into gyral based regions of interest. Neurolmage 31 , 968-980 (2006).

Klein, A. \& Tourville, J. 101 Labeled Brain Images and a Consistent Human Cortical Labeling Protocol. Front. Neurosci. 6, (2012).

Klein, A. et al. Mindboggling morphometry of human brains. PLoS Comput Biol 13, e1005350 (2017)

Open Science Framework: https://osf.io/nhtur/

Harvard Dataverse: $h$ ttps://dataverse. harvard edu/dataverse/mindboggle

Labels: https://mindboggle. readthedocs.io/en/latest/labels.html

Github: https://github.com/nipy/mindboggle

http://www.neuromorphometrics.com/

https://surfer.nmr.mgh.harvard.edu/

Gordon, E. M. et al. Generation and Evaluation of a Cortical Area Parcellation from Resting-State Correlations. Cereb. Cortex 26, 288-303 (2016).

https://sites.wustl.edu/petersenschlaggarlab/resources/

Hammers, A et al. Three-dimensional maximum probability atlas of the human

brain, with particular reference to the temporal lobe. Hum. Brain Mapp. 19, 224-247 (2003).

Gousias, I. S. et al. Automatic segmentation of brain MRls of 2-year-olds into 83 regions of interest. Neurolmage 40,672-684 (2008).

http://brain-development.org/brain-atlases/adult-brain-atlases/adult-brain-maximumprobability-map-hammers-mith-atlas-n30r83-in-mni-space/

https.//fsl.fmrib.ox.ac.uk/fsl/fslwiki/Atlases

FreeSurfer description about CMA: http://freesurfer.net/fswiki/CMA

Link to website archive: https://web.archive.org/web/20180413052010/http://www. cma.mgh.harvard.edu/

Wakana, S. et al. Reproducibility of quantitative tractography methods applied to cerebral white matter. Neurolmage 36, 630-644 (2007).

Hua, K. et al. Tract probability maps in stereotaxic spaces: Analyses of white matter anatomy and tract-specific quantification. Neurolmage 39, 336-347 (2008).

MRI atlas of human white matter. (Elsevier, Acad. Press, 2011)

(1) Amunts, K., Mohlberg, H., Bludau, S. \& Zilles, K. Julich-Brain: A 3D probabilistic atlas of the human brain's cytoarchitecture. 6 (2020).

(2) Eickhoff, S. B. et al. A new SPM toolbox for combining probabilistic cytoarchitectonic maps and functional imaging data. Neurolmage 25, 1325-1335 (2005)

https://www.fz-juelich.de/inm/inm-1/DE/Forschung/_docs/SPMAnatomyToolbox/ SPMAnatomyToolbox_node.html

Glasser, M. F. et al. A multi-modal parcellation of human cerebral cortex. Nature $536,171-178(2016)$.

Coalson, T. S., Van Essen, D. C. \& Glasser, M. F. The impact of traditional neuroimaging methods on the spatial localization of cortical areas. Proc Natl Acad Sci USA 115, E6356-E6365 (2018).

https://balsa.wustl.edu/

Zalesky, A. et al. Whole-brain anatomical networks: does the choice of nodes matter? Neuroimage 50, 970-83 (2010)

(1) Goni, J. et al. Resting-brain functional connectivity predicted by analytic measures of network communication. Proceedings of the National Academy of Sciences 111, 833-838 (2014).

(2) Mišić, B. et al. Cooperative and Competitive Spreading Dynamics on the Human Connectome. Neuron 86, 1518-29 (2015).

Table S1. (cont.) Atlas sources and references. | This table provides a short note and references to the source material of common atlases in the neuroscience literature. See also Table 1. 
bioRxiv preprint doi: https://doi.org/10.1101/2021.06.11.448063; this version posted October 7, 2021. The copyright holder for this preprint (which was not certified by peer review) is the author/funder, who has granted bioRxiv a license to display the preprint in perpetuity. It is made available under aCC-BY-NC 4.0 International license.

\begin{tabular}{|c|c|c|c|}
\hline Atlas & Source & Note & Reference(s) \\
\hline MN & 41 & $\begin{array}{l}\text { Included with FSL. See website for further details. Included } \\
\text { structures are (1) Caudate, (2) Putamen, (3) Thalamus, (4) } \\
\text { Insula, (5) Frontal lobe, (6) Temporal lobe, (7) Parietal lobe, (8) } \\
\text { Occipital lobe, and (9) Cerebellum. }\end{array}$ & $\begin{array}{l}\text { (1) Website: http://www.talairach.org/about.html } \\
\text { (2) http://www.talairach.org/about.html } \\
\text { (3) Mazziotta, J. et al. A probabilistic atlas and reference system for the human } \\
\text { brain: International Consortium for Brain Mapping (ICBM). Phil. Trans. R. Soc. } \\
\text { Lond. B 356, 1293-1322 (2001). }\end{array}$ \\
\hline \multirow[t]{2}{*}{ Schaefer } & 42 & Original publication about functional parcellations. & $\begin{array}{l}\text { Schaefer, A. et al. Local-Global Parcellation of the Human Cerebral Cortex from } \\
\text { Intrinsic Functional Connectivity MRI. Cerebral Cortex 28, 3095-3114 (2018). }\end{array}$ \\
\hline & 43 & Github and detailed documentation of atlases. & $\begin{array}{l}\text { https://github.com/ThomasYeoLab/CBIG/tree/master/stable_projects/brain_parcel- } \\
\text { lation/Schaefer2018_LocalGlobal }\end{array}$ \\
\hline \multirow[t]{5}{*}{ Talairach } & 44 & Download: Included with FSL. Also available through website. & Website: http://www.talairach.org/ \\
\hline & 45 & $\begin{array}{l}\text { The anatomical region labels were electronically derived from } \\
\text { axial sectional images in the } 1988 \text { Talairach Atlas. The atlas was } \\
\text { digitized and manually traced into a volume-occupant hierarchy } \\
\text { of anatomical regions detailed these publications (i.e. the pages } \\
\text { of the } 1988 \text { textbook with drawings were photocopied and } \\
\text { transformed into the computerized coordinate system). }\end{array}$ & $\begin{array}{l}\text { (1) Lancaster, J. L., Evans, A. C. \& Toga, A. W. Automated Labeling of the Human } \\
\text { Brain: A Preliminary Report on the Development and Evaluation of a For- } \\
\text { ward-Transform Method. 238-242 (1997). } \\
\text { (2) Lancaster, J. L. et al. Automated Talairach Atlas Labels For Functional Brain } \\
\text { Mapping. 120-131 (2000). }\end{array}$ \\
\hline & 46 & $\begin{array}{l}\text { (1) First atlas in } 1957 \text { focusing on the subcortical deep gray } \\
\text { nucelli, (2) second atlas in } 1967 \text { focusing on the telencepha- } \\
\text { lon, (3) third atlas in } 1988 \text { focusing on the whole brain. Most } \\
\text { researchers preferred the use of the Talairach atlas to report } \\
\text { the localization of the activations detected in functional imaging } \\
\text { studies because it offers a detailed anatomical brain description } \\
\text { within the stereotaxic space, including Brodmann's areas. }\end{array}$ & $\begin{array}{l}\text { (1) Talairach, J., David, M., Tournoux, P., Corredor, H. \& Kvasina, T. Atlas d'Anato- } \\
\text { mie Stéréotaxique. Repérage Radiologique Indirect des Noyaux Gris Centraux des } \\
\text { Régions Mésencephalosousoptique et Hypothalamique de l'Homme. (1957). } \\
\text { (2) Talairach, J. \& Szikla, G. Atlas of Stereotaxic Anatomy of the Telencephalon. } \\
\text { (Masson, 1967) } \\
\text { (3) Talairach, J. \& Tournoux, P. Co-planar stereotaxic atlas of the human brain: } \\
\text { 3-dimensional proportional system: an approach to cerebral imaging. (Georg } \\
\text { Thieme, 1988). }\end{array}$ \\
\hline & 47 & Historical publication about Jean Talairach. & $\begin{array}{l}\text { Harary, M. \& Cosgrove, G. R. Jean Talairach: a cerebral cartographer. Neurosurgi- } \\
\text { cal Focus 47, E12 (2019). }\end{array}$ \\
\hline & 48 & Comparison between $\mathrm{MNI}$ and Talairach Coordinates. & $\begin{array}{l}\text { Lancaster, J. L. et al. Bias between MNI and Talairach coordinates analyzed using } \\
\text { the ICBM-152 brain template. Hum. Brain Mapp. } 28,1194-1205 \text { (2007). }\end{array}$ \\
\hline \multirow[t]{2}{*}{ Yeo } & 49 & Original publication about functional parcellations. & $\begin{array}{l}\text { Thomas Yeo, B. T. et al. The organization of the human cerebral cortex estimated } \\
\text { by intrinsic functional connectivity. Journal of Neurophysiology 106, 1125-1165 } \\
\text { (2011) }\end{array}$ \\
\hline & 50 & Website from FreeSurfer. & https://surfer.nmr.mgh.harvard.edu/fswiki/CorticalParcellation_Ye02011 \\
\hline \multirow[t]{4}{*}{ Region-specific } & 51 & Thalamus - based on ex vivo analysis. & $\begin{array}{l}\text { Iglesias, J. E. et al. A probabilistic atlas of the human thalamic nuclei combining ex } \\
\text { vivo MRI and histology. Neurolmage } 183,314-326(2018) \text {. }\end{array}$ \\
\hline & 52 & Hippocampus - based on ex vivo analysis. & $\begin{array}{l}\text { Iglesias, J. E. et al. A computational atlas of the hippocampal formation using ex } \\
\text { vivo, ultra-high resolution MRI: Application to adaptive segmentation of in vivo MRI. } \\
\text { Neurolmage } 115,117-137 \text { (2015). }\end{array}$ \\
\hline & 53 & Structural atlas of Cerebellum. Included with FSL. & $\begin{array}{l}\text { Diedrichsen, J., Balsters, J. H., Flavell, J., Cussans, E. \& Ramnani, N. A probabilis- } \\
\text { tic MR atlas of the human cerebellum. Neurolmage } 46,39-46 \text { (2009). }\end{array}$ \\
\hline & 54 & Functional atlas of Cerebellum. & $\begin{array}{l}\text { (1) Xue, A. et al. The Detailed Organization of the Human Cerebellum Estimated by } \\
\text { Intrinsic Functional Connectivity Within the Individual. } 69 \text {. } \\
\text { (2) Buckner, R. L., Krienen, F. M., Castellanos, A., Diaz, J. C. \& Yeo, B. T. T. The } \\
\text { organization of the human cerebellum estimated by intrinsic functional connectivity. } \\
\text { Journal of Neurophysiology 106, 2322-2345 (2011). } \\
\text { (2) GitHub: https://github.com/ThomasYeoLab/CBIG/tree/master/stable_projects/ } \\
\text { brain_parcellation/Xue2021_IndCerebellum }\end{array}$ \\
\hline \multirow[t]{2}{*}{ Population-specific } & 55 & Pediatric/Neonatal. & $\begin{array}{l}\text { Alexander, B. et al. A new neonatal cortical and subcortical brain atlas: the Mel- } \\
\text { bourne Children's Regional Infant Brain (M-CRIB) atlas. Neurolmage 147, 841-851 } \\
\text { (2017). }\end{array}$ \\
\hline & 56 & Disease-specific: example of a multiple sclerosis lesional atlas. & Sahraian, M. A. \& Radue, E.-W. MRI atlas of MS lesions. (Springer, 2008). \\
\hline
\end{tabular}

Table S1. (cont.) Atlas sources and references. | This table provides a short note and references to the source material of common atlases in the neuroscience literature. See also Table 1. 
bioRxiv preprint doi: https://doi.org/10.1101/2021.06.11.448063; this version posted October 7, 2021. The copyright holder for this preprint (which was not certified by peer review) is the author/funder, who has granted bioRxiv a license to display the preprint in perpetuity. It is made available under aCC-BY-NC 4.0 International license.

\section{Atlas Morphology: Sizes and Shapes}

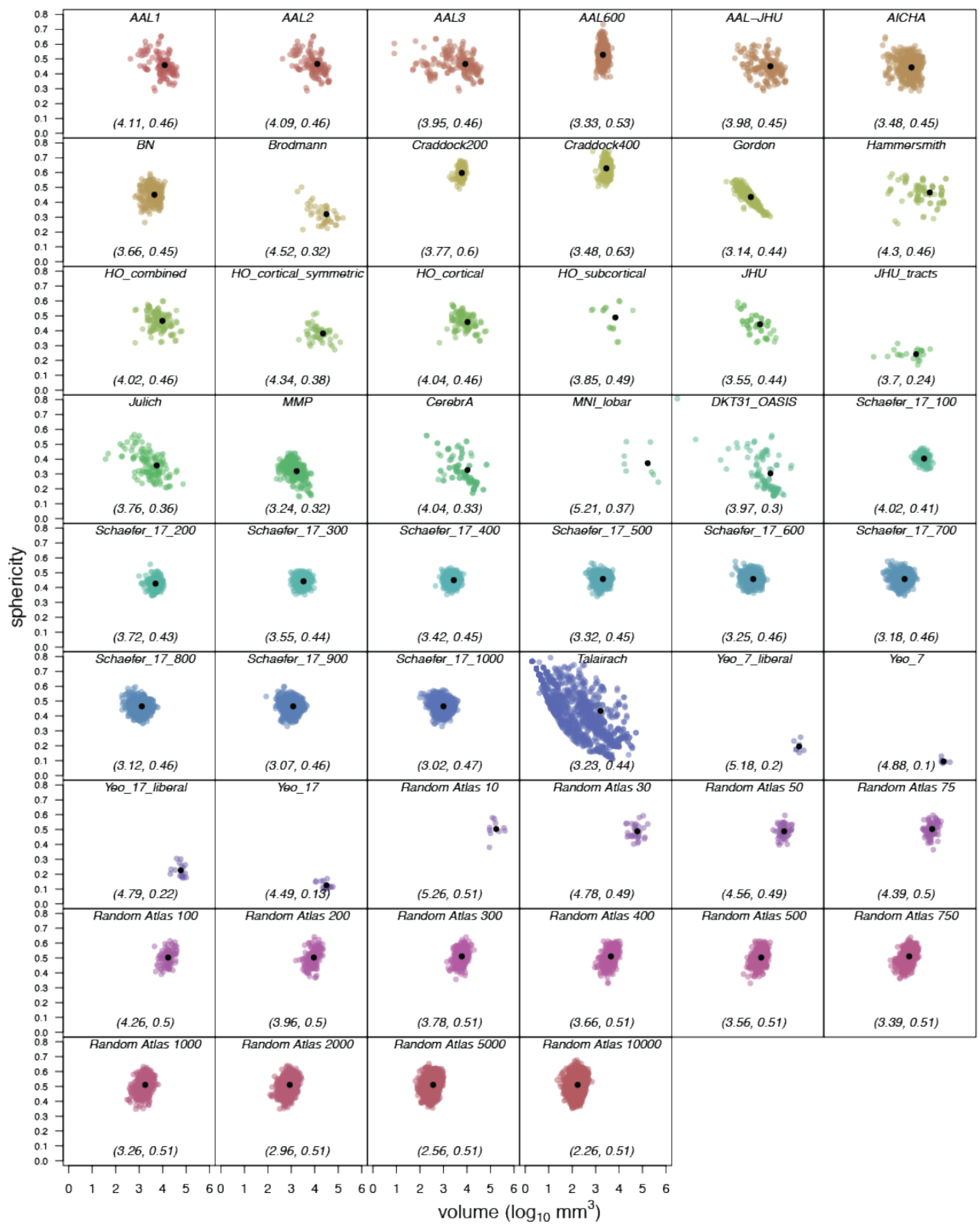

Fig. S2. Atlas Morphology: Sizes and Shapes. | All standard atlases and one permutation for each of the standard atlases are shown here. Volume means and sphericity means are in parentheses at the bottom of each graph. See Table S1 for atlas abbreviations, descriptions, and sources. 


\section{Remaining atlases (Repeat of Fig. 3)}
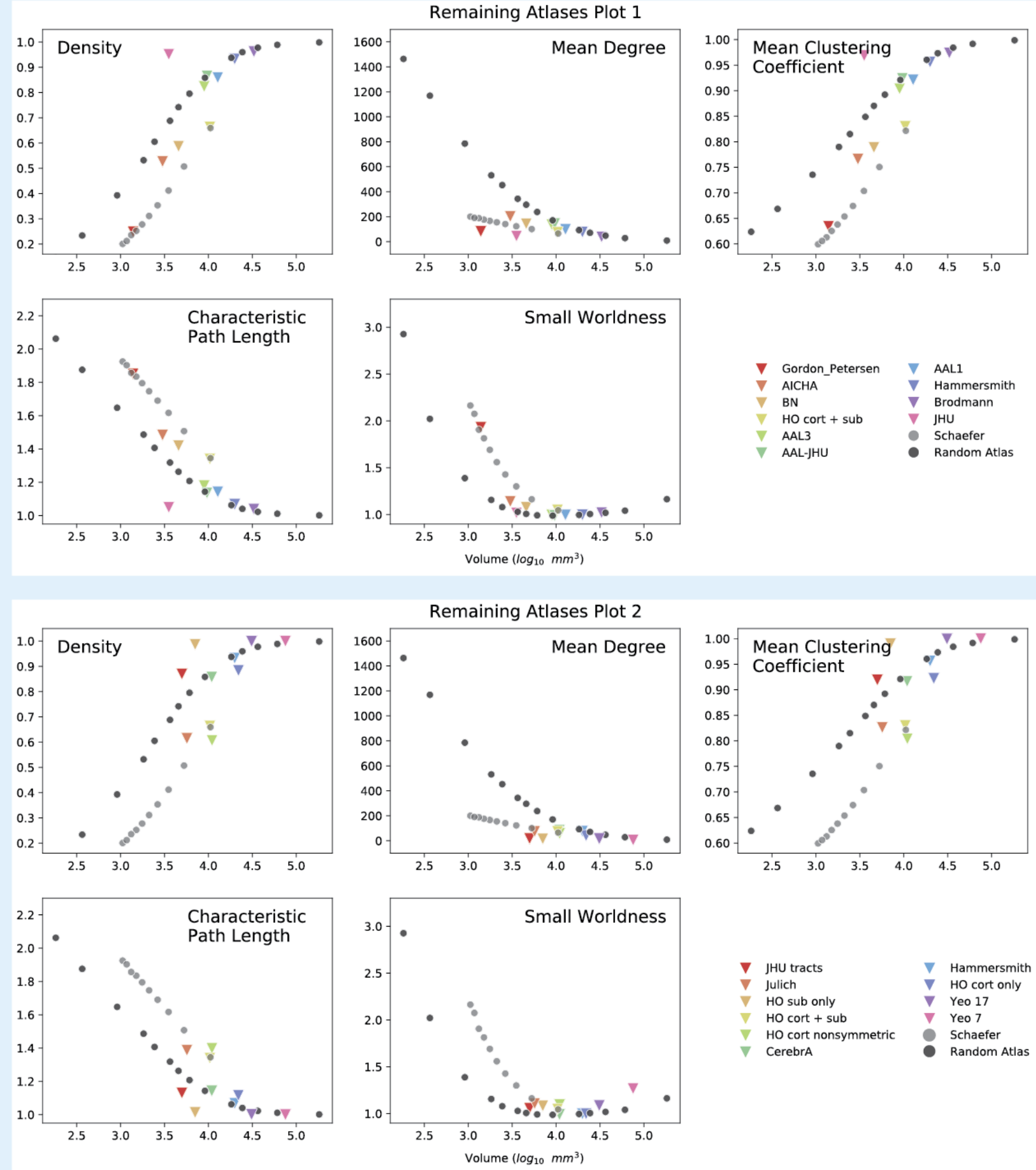

Fig. S3. Structure-Function Correlation (SFC) for All Atlases. | We show network measures the remaining atlases illustrated in Table 2. See Table S1 for atlas descriptions. HO, Harvard-Oxford; Sub, subcortical; Cort, cortical 


\section{Controls and patients separated (Repeat of Fig. 3)}
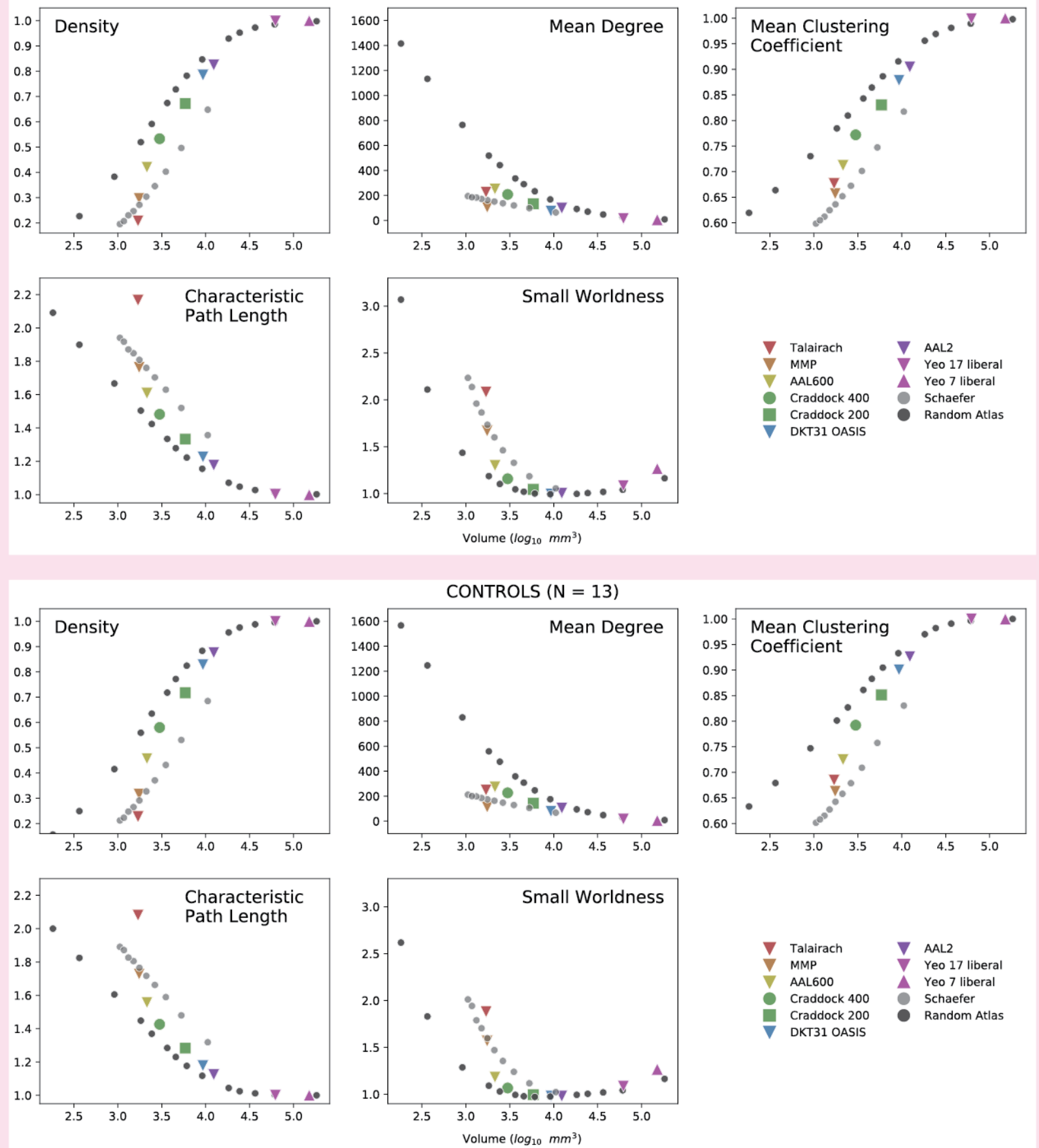

Fig. S4. Network Measures: Controls vs Patients. | We replicate Fig. $2(\mathrm{~N}=41)$ in the manuscript by separating out controls $(\mathrm{N}=13)$ and patients $(\mathrm{N}=28)$. All global network measures above are similar between patients and controls, with patients having slightly lower (but not significant, Fig. 2 bottom right panel) measurements for the different network properties. Specific connectivity differences between controls and patients were not explored (e.g. to explore if connections from the hippocampus to the anterior cingulate are changed in temporal lobe epilepsy) and out of the scope of this manuscript. See Table S1 for atlas descriptions. 


\section{Re-calculating network measures at different thresholds (Repeat of Fig. 3)}
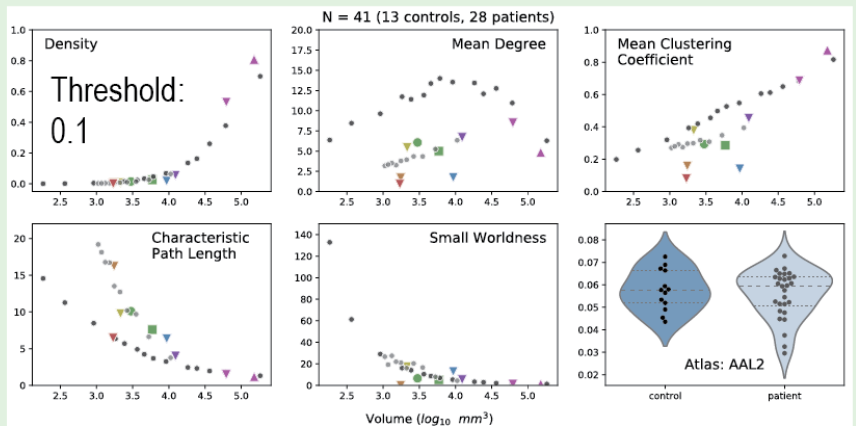

Volume $\left(\log _{10} \mathrm{~mm}^{3}\right)$
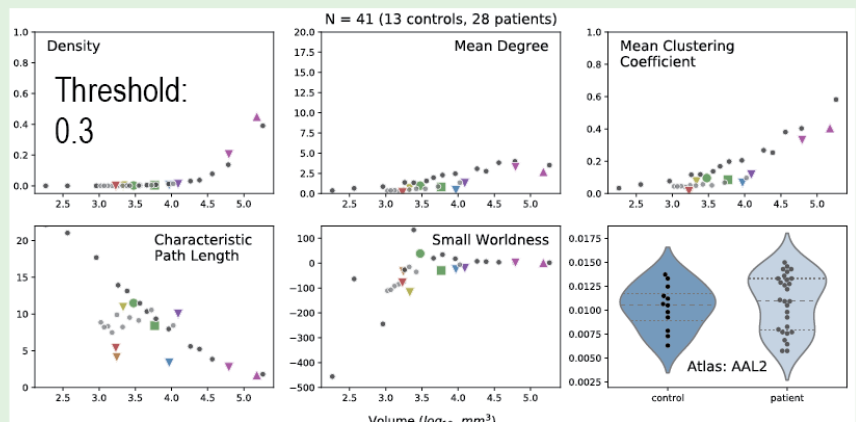

Volume $\left(\log _{10} \mathrm{~mm}^{3}\right)$

$\nabla$ Talairach

$\nabla$ AAL2

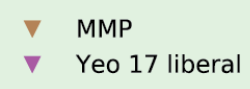

$\nabla \quad$ AAL600

A Yeo 7 liberal
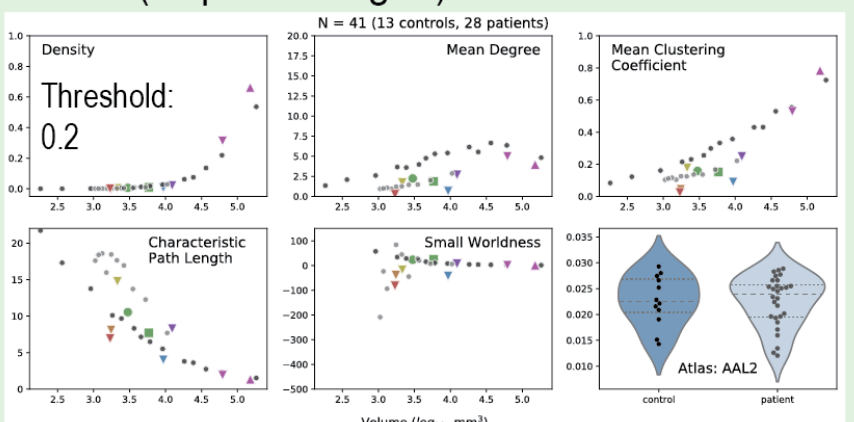

Volume $\left(\log _{10} \mathrm{~mm}^{3}\right)$
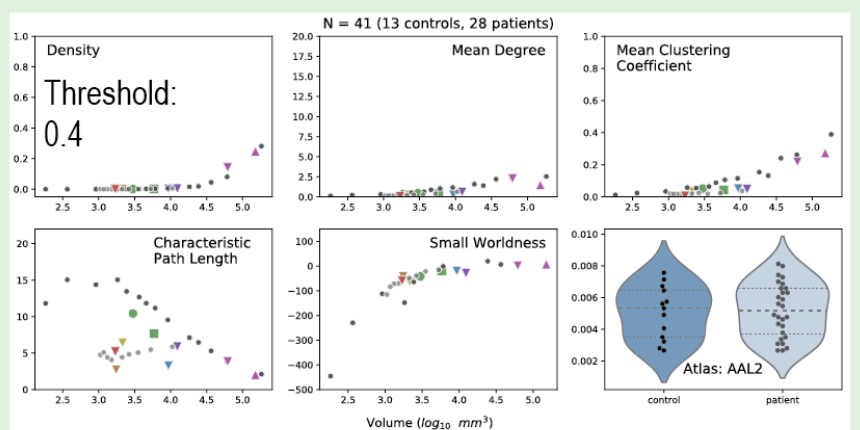

- Craddock 400

- Craddock 200

- Random Atlas

Fig. S5. Network Measures: different thresholds. | We replicate Fig. $2(\mathrm{~N}=41)$ in the manuscript by calculating network measures using different thresholds. The main text figure includes all weights with no threshold (threshold $=0)$. We set thresholds at 01., 0.2, 0.3, and 0.4. This was done to show how various network measures may also change when eliminating low-level connections at different thresholds. 
bioRxiv preprint doi: https://doi.org/10.1101/2021.06.11.448063; this version posted October 7, 2021. The copyright holder for this preprint (which was not certified by peer review) is the author/funder, who has granted bioRxiv a license to display the preprint in perpetuity. It is made available under aCC-BY-NC 4.0 International license.
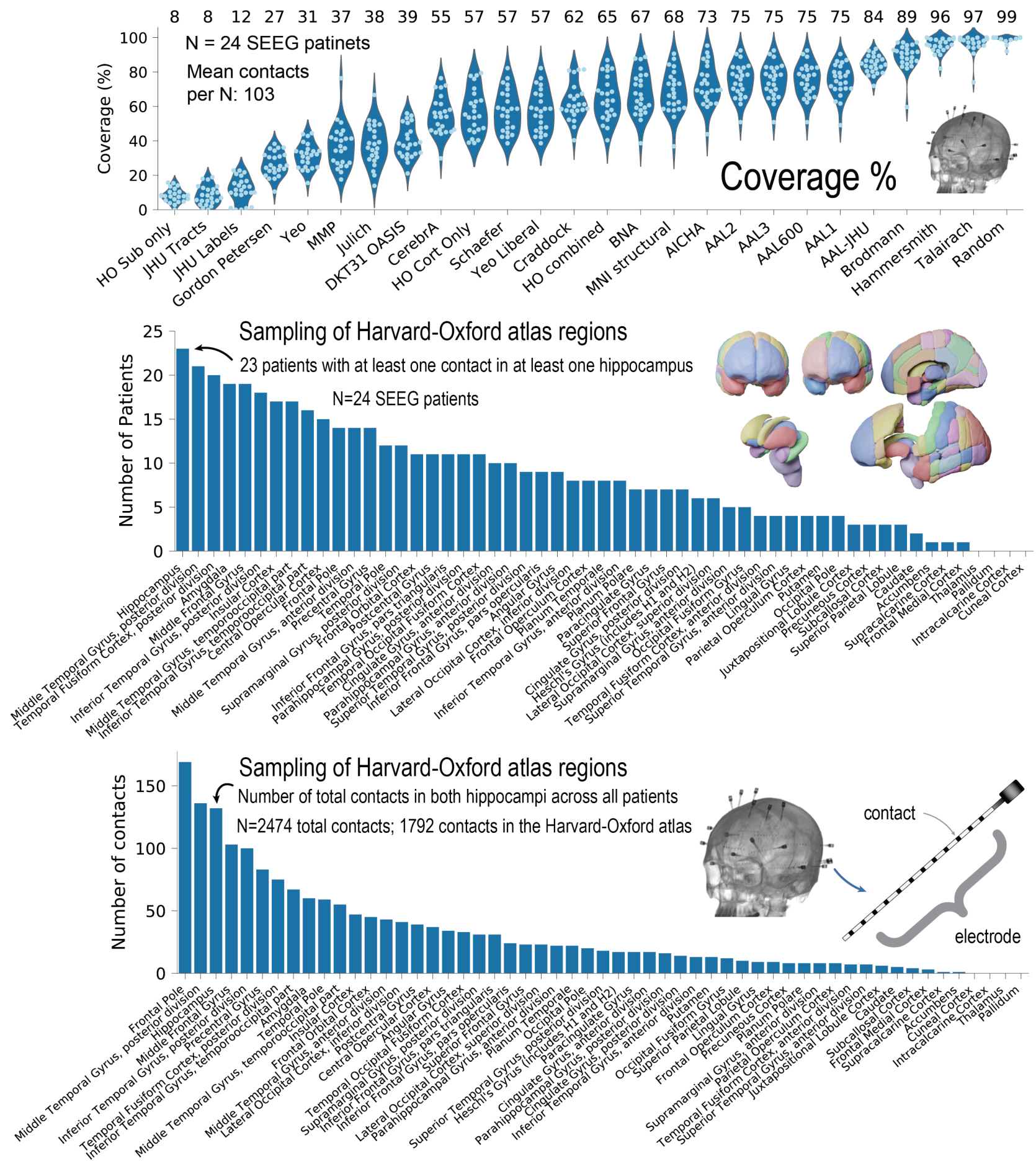

Fig. S6. Coverage of electrode contacts. | Top: We show the percentage of contacts assigned a region given an atlas. If a contact fell outside an atlas, it would not be assigned a location and would not be used in SFC analysis. We also show the Harvard-Oxford atlas regions (cortical and subcortical combined) that contain electrode contacts (middle and bottom figures). The middle figure shows the number of patients with at least one contact in an atlas region (at least one of the regions on both hemispheres). The bottom figure shows the total number of contacts in each listed region. Note that 1792 out of 2474 contacts $(72 \%)$ contained within the brain parenchyma (gray matter or white matter) is higher than the mean percent coverage listed in the top figure ( $65 \%$ for the HO combined) because some patients with fewer contacts may have lower coverage by the atlas, thus bringing the mean percent down. Also note the larger number of contacts in the frontal pole because this region in the Harvard-Oxford atlas is large. We chose to show the Harvard-Oxford atlas because it has the largest effect size in Fig. 6. 


\section{Results for "Brain Atlas" per 100,000 citations in PubMed proportion for each search by year, 1945 to 2020 \\ 60}

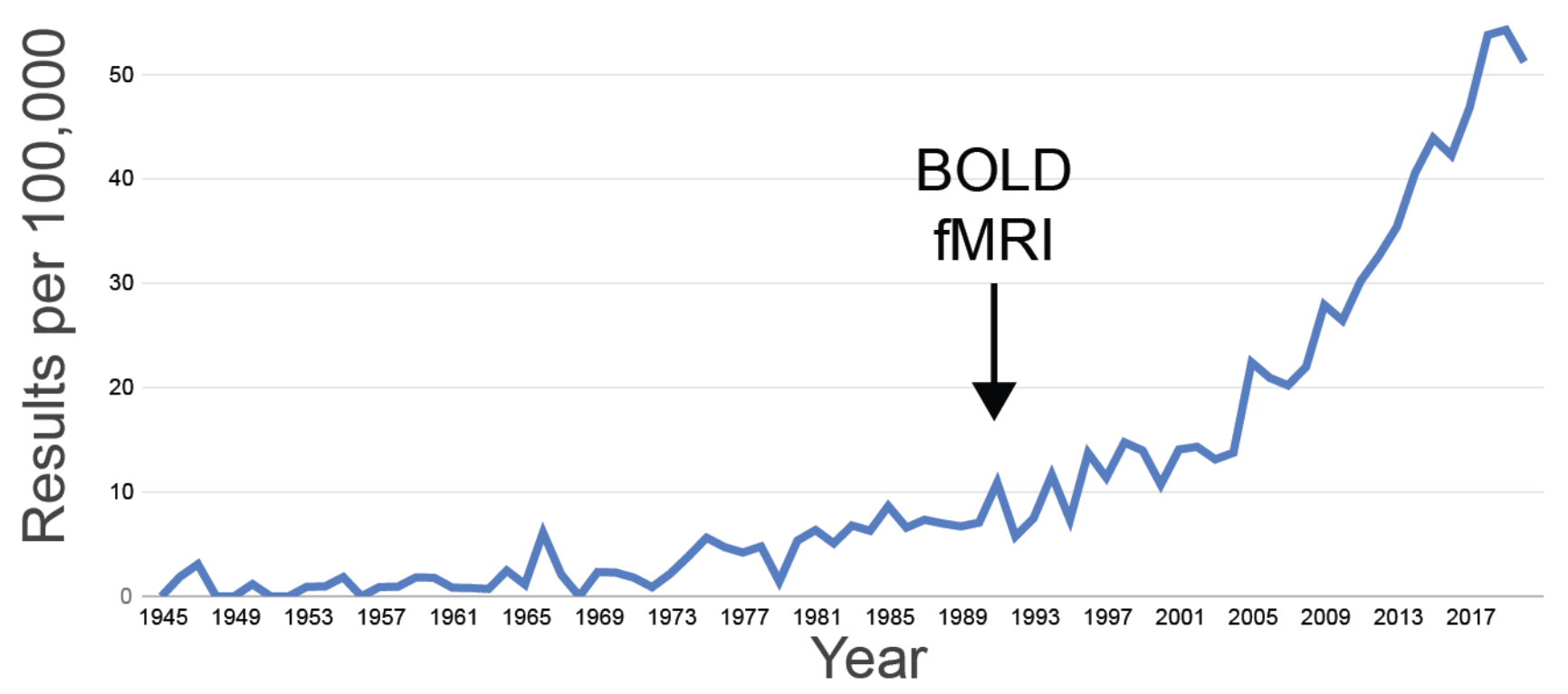

Fig. S7. The increase in publications related to brain atlases. | We searched for any publications since 1945 using the term "Brain Atlas" on PubMed. We note that since the introduction of BOLD fMRI in 1990, the need for neuroanatomical maps of the brain has increased, especially in the neuroimaging community. Many atlases have been published over the last 30 years, and many publications across the neuroscience literature have used these atlases. However, no comprehensive study exists evaluating, in any regard, to the suitability and nuances related to these atlases. We hope our work provides a valuable resource to others in our field, launches a larger discussion to critically evaluating the neuroanatomy of the brain, and direct future reproducible research for other scientists and clinician investigators. Due to space constraints in Figure 7d, atlases illustrated are listed from left/right, top/bottom: AAL1, Schaefer, MMP, AICHA, Yeo, BNA, Julich, Random 10k and 10, Harvard-Oxford Cortical, DKT + Freesurfer aseg, JHU labels, AAL + JHU labels combined, Harvard-Oxford, Talairach, Macaque, Cerebellum, M-CRIB, Yeo 17 liberal, Yeo 17 conservative, Random 30 hard and soft, DKT, BNA, Random 100 parcellations. 


\begin{tabular}{|c|c|c|c|c|c|c|}
\hline Patient & Age & Sex & Localization: suspected seizure onset zone & Control & Age & Sex \\
\hline sub-patient01 & 58 & M & Poorly localized. R temporal interictal activity. & sub-control01 & 24 & $\mathrm{M}$ \\
\hline sub-patient02 & 28 & $\mathrm{~F}$ & $\mathrm{~L}$ anterior temporal lobe & sub-control02 & 40 & $\mathrm{~F}$ \\
\hline sub-patient03 & 27 & $\mathrm{~F}$ & L hippocampus and amygdala & sub-control03 & 31 & $\mathrm{M}$ \\
\hline sub-patient 04 & 20 & $\mathrm{~F}$ & L basal ganglia infarct & sub-control04 & 29 & $\mathrm{M}$ \\
\hline sub-patient $05^{\star *}$ & 36 & M & $\mathrm{R}$ frontal arteriovenous malformation & sub-control05 & 40 & $\mathrm{M}$ \\
\hline sub-patient06 & 57 & $\mathrm{~F}$ & Poorly localized. Possibly bitemporal onset & sub-control06 & 48 & $\mathrm{~F}$ \\
\hline sub-patient $07^{\star *}$ & 37 & M & L temporal lobe/hippocampus/amygdala & sub-control07 & 22 & $\mathrm{M}$ \\
\hline sub-patient $08^{\star *}$ & 34 & M & $\mathrm{R}$ frontal, anterior cingulate gyrus & sub-control08 & 35 & $\mathrm{~F}$ \\
\hline sub-patient $09^{* *}$ & 47 & $\mathrm{~F}$ & L hippocampus & sub-control09 & 27 & $\mathrm{~F}$ \\
\hline sub-patient 10 & 42 & $\mathrm{~F}$ & $\mathrm{R}$ temporal lobe/L temporal lobe & sub-control10 & 67 & $\mathrm{~F}$ \\
\hline sub-patient11 & 27 & M & L hippocampus, then amygdala & sub-control11 & 33 & $\mathrm{~F}$ \\
\hline sub-patient12 & 35 & M & Poorly localized. Possibly multifocal epilepsy & sub-control12 & 27 & M \\
\hline sub-patient $13^{\star *}$ & 36 & $\mathrm{~F}$ & L temporal & sub-control13 & NR & NR \\
\hline sub-patient $14^{\star *}$ & 29 & $\mathrm{~F}$ & L superior Frontal Sulcus & & & \\
\hline sub-patient15 & 33 & $\mathrm{~F}$ & L mesial temporal lobe & & & \\
\hline sub-patient16 & 29 & M & Poorly localized. Possibly multifocal epilepsy & & & \\
\hline sub-patient17 & 31 & $\mathrm{~F}$ & L mesial temporal lobe & & & \\
\hline sub-patient $18^{\star *}$ & 26 & $\mathrm{~F}$ & L heterotopia, left hippocampus & & & \\
\hline sub-patient $19^{\star *}$ & 23 & M & L temporal/posterior lateral neocortical & & & \\
\hline sub-patient 20 & 30 & M & L temporal encephalomalacia & & & \\
\hline sub-patient 21 & 24 & M & $\mathrm{R}$ anterior temporal lobe & & & \\
\hline sub-patient 22 & 59 & $\mathrm{~F}$ & $\mathrm{R}$ frontal-parietal lobe & & & \\
\hline sub-patient 23 & 28 & $\mathrm{~F}$ & $\mathrm{~L}$ or $\mathrm{R}$ superior temporal gyrus & & & \\
\hline sub-patient $24^{\star *}$ & 47 & $\mathrm{~F}$ & $\mathrm{R}$ anterior temporal & & & \\
\hline sub-patient 25 & 40 & $\mathrm{~F}$ & L temporal lobe near Heschl's gyrus & & & \\
\hline sub-patient 26 & 37 & $\mathrm{~F}$ & $\mathrm{~L}$ amygdala/anterior temporal pole & & & \\
\hline sub-patient 27 & 30 & M & $\mathrm{L}$ amygdala/hippocampus & & & \\
\hline sub-patient $28^{\star *}$ & 28 & M & L mesial temporal lobe & & & \\
\hline
\end{tabular}

Table S2. Patient and control demographics.| Patient IDs with asterisk have clinically annotated seizures for structurefunction calculation. Localization of the seizure onset zone was pulled from patient charts, either from the clinically hypothesized brain regions if the patient did not undergo surgery, or if the patient underwent surgery, the targeted location for resection or ablation. One control did not have age or sex information. M, Male; F: Female; L, left; R, Right; NR, Not reported 
AAL
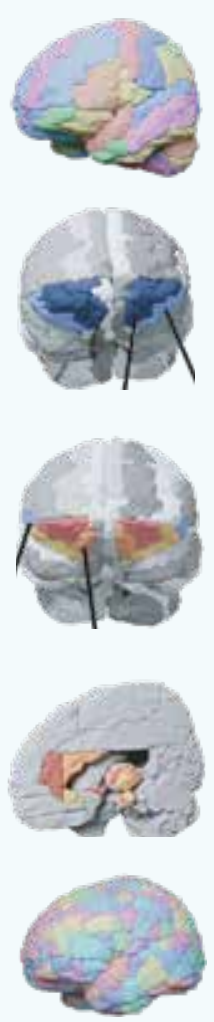

AICHA

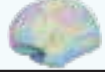

Brainnetome
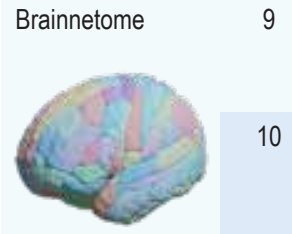

8

AICHA tries to account for homotopy: the concept that each region in one hemisphere has a homologue in the other.

9 Connectivity-based atlas. Further subdivision of structural parcellations using the DK (Desikan-Killiany) protocol, with adjustments.

10 DSI studio created by Fang-Cheng (Frank) Yeh. Many reconstruction and tracking algorithms are published and incorporated into DSI Studio. See citations page on website. Many atlases available, including Brainnetome. Can use custom atlas.

Brodmann

12

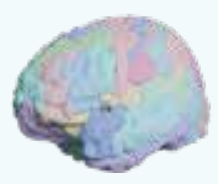

13

The atlas is available through MRIcro, a legacy tool developed by Chris Rorden (University of South Carolina). The atlas is based on work from the Van Essen lab (Washington University in St. Louis) with corresponding Talairach coordinates, and transformed by Krish Singh (Cardiff University) to MNI space.

\section{Reference(s)}

(1) Tzourio-Mazoyer, N. et al. Automated Anatomical Labeling of Activations in SPM Using a Macroscopic Anatomical Parcellation of the MNI MRI Single-Subject Brain. Neurolmage 15, 273-289 (2002).

(2) Collin-27 template: Holmes, C. J. et al. Enhancement of MR Images Using Registration for Signal Averaging: Journal of Computer Assisted Tomography 22, 324-333 (1998).

(3) Website about Collin-27: https://www.bic.mni.mcgill.ca/ServicesAtlases/Colin27

(1) Rolls, E. T., Joliot, M. \& Tzourio-Mazoyer, N. Implementation of a new parcellation of the orbitofrontal cortex in the automated anatomical labeling atlas. Neurolmage 122, 1-5 (2015).

(2) Chiavaras, M. M. \& Petrides, M. Orbitofrontal sulci of the human and macaque monkey brain. The Journal of Comparative Neurology 422, 35-54

(3) Dejerine, J. Anatomie des centres nerveux. (Rueff Paris, 1895).

Rolls, E. T., Huang, C.-C., Lin, C.-P., Feng, J. \& Joliot, M. Automated anatomical labelling atlas 3. Neurolmage 206, 116189 (2020).

https://www.gin.cnrs.fr/en/tools/aal/

(1) Statistical parametric mapping: the analysis of functional brain images. (Elsevier/Academic Press, 2007).

(2) Website: https://www.fil.ion.ucl.ac.uk/spm/ext/

Bassett, D. S., Brown, J. A., Deshpande, V., Carlson, J. M. \& Grafton, S. T. Conserved and variable architecture of human white matter connectivity. Neurolmage 54, 1262-1279 (2011)

(1) Ashourvan, A., Telesford, Q. K., Verstynen, T., Vettel, J. M. \& Bassett, D. S Multi-scale detection of hierarchical community architecture in structural and functional brain networks. (2017)

(2) Hermundstad, A. M. et al. Structurally-Constrained Relationships between Cognitive States in the Human Brain. PLoS Comput Biol 10, e1003591 (2014).

Joliot, M. et al. AICHA: An atlas of intrinsic connectivity of homotopic areas. Journal of Neuroscience Methods 254, 46-59 (2015)

Fan, L. et al. The Human Brainnetome Atlas: A New Brain Atlas Based on Connectional Architecture. Cerebral cortex (New York, N.Y. : 1991) 26, 3508-26 (2016). Website: http://atlas.brainnetome.org

(1) Website: http://dsi-studio.labsolver.org/

(2) Example of reconstruction method: Fang-Cheng Yeh, Wedeen, V. J. \& Tseng, W.-Y. I. Generalized q-Sampling Imaging. IEEE Trans. Med. Imaging 29, 1626-1635 (2010).

Zilles, K. \& Amunts, K. Centenary of Brodmann's map - conception and fate. Nat Rev Neurosci 11, 139-145 (2010

(1) Original German: Vergleichende Lokalisationslehre der Grosshirnrinde in ihren Prinzipien dargestellt auf Grund des Zellenbaues. (1909)

(2) English translation: Brodmann, K. \& Gary, L. J. Brodmann's localisation in the cerebral cortex: the principles of comparative localisation in the cerebral cortex based on cytoarchitectonics. (Springer, 2006

(1) Chris Rorden legacy tools webpage: https://people.cas.sc.edu/rorden/

(2) Updated webpage: https://crnl.readthedocs.io/

(3) About Brodmann atlas: https://people.cas.sc.edu/rorden/mricro/lesion.html

(4) BALSA: https://balsa.wustl.edu/Wz8r

Manera, A. L., Dadar, M., Fonov, V. \& Collins, D. L. CerebrA, registration and manual label correction of Mindboggle-101 atlas for MNI-ICBM152 template. Sci Data 7, 237 (2020).

Website: https://doi.gin.g-node.org/10.12751/g-node.be5e62

Craddock, R. C., James, G. A., Holtzheimer, P. E., Hu, X. P. \& Mayberg, H. S. A whole brain $\mathrm{fMRI}$ atlas generated via spatially constrained spectral clustering. Hum. Brain Mapp. 33, 1914-1928 (2012).

GitHub: http://ccraddock.github.io/cluster_roi/atlases.html

ABIDE: http://preprocessed-connectomes-project.org/abide/Pipelines.html 

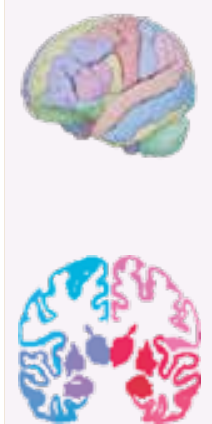

23 FreeSurfer.

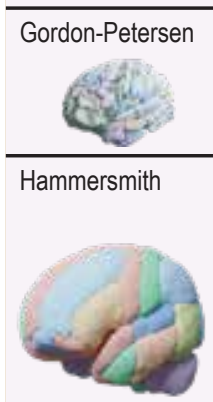

24 Original article.

25 Resource to download atlas.

26 Original article (for regions 1-49), including their Hammersmith protocol (or "algorithm").

27 Updated regions (for regions 50-83).

28 Download atlas with 83 regions.

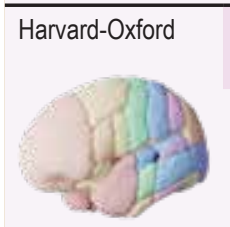

Atlas developed at the Center for Morphometric Analysis (CMA) at Massachusetts General Hospital and distributed with FSL.

30 Individual segmentations were segmented by CMA using inhouse software. Probability maps were then created. Freesurfer link (right) has archived CMA's website and contains the Harvard-Oxford labeling protocols.

JHU

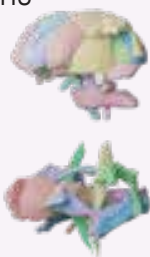

31

JHU labels: Protocol to reconstruct eleven white matter tracts and their segmentation into ROI labels. Included in FSL.

32 JHU Tracts: white matter parcellation atlas based on DTI probabilistic tractography of 11 major white matter tracts $\mathrm{d}$. Protocol defining manually identified ROls from which the tracts were formed are described in Wakana et al. (2005). Included in FSL.

33 Textbook with more information about these atlases.

34 Cytoarchitecture map. Successor to both the Brodmann and Eickhoff-Zilles atlases. The Eichoff-Zilles is an SPM toolbox (see note is source 5 about the AAL atlas) for probabilistic cytoarchitecture.

35 Website for the Julich Atlas and SPM toolbox.

36 Original article on multi-modal approach.

37 Information on surface vs volume based methodologies for localization of neuroanatomy.

38 Website to download data. Volumetric version also included in DSI-studio. Note the volume note above.

Random

39 Random atlas algorithm (pseudo-grassfire algorithm).

$40 \quad$ Use case of random atlas. Goni et al. (2014) study the structure-function relationship in the brain with tractography and fMRI. They used random cortical atlases of 1170 equally sized regions. Misic et al. (2015) used random cortical atlases of 1015 equally sized regions.

\section{Reference(s)}

Desikan, R. S. et al. An automated labeling system for subdividing the human cerebral cortex on MRI scans into gyral based regions of interest. Neurolmage 31, 968-980 (2006).

Klein, A. \& Tourville, J. 101 Labeled Brain Images and a Consistent Human Cortical Labeling Protocol. Front. Neurosci. 6, (2012).

Klein, A. et al. Mindboggling morphometry of human brains. PLoS Comput Biol 13, e1005350 (2017)

Open Science Framework: https://osf.io/nhtur/

Harvard Dataverse: https://dataverse.harvard.edu/dataverse/mindboggle

Labels: https://mindboggle.readthedocs.io/en/latest/labels.html

GitHub: https://github.com/nipy/mindboggle

http://www.neuromorphometrics.com/

https://surfer.nmr.mgh.harvard.edu/

Gordon, E. M. et al. Generation and Evaluation of a Cortical Area Parcellation from Resting-State Correlations. Cereb. Cortex 26, 288-303 (2016).

https://sites.wustl.edu/petersenschlaggarlab/resources/

Hammers, A. et al. Three-dimensional maximum probability atlas of the human brain, with particular reference to the temporal lobe. Hum. Brain Mapp. 19, 224-247 (2003).

Gousias, I. S. et al. Automatic segmentation of brain MRIs of 2-year-olds into 83 regions of interest. Neurolmage 40, 672-684 (2008).

http://brain-development.org/brain-atlases/adult-brain-atlases/adult-brain-maximumprobability-map-hammers-mith-atlas-n30r83-in-mni-space/

https://fsl.fmrib.ox.ac.uk/fsl/fslwiki/Atlases

FreeSurfer description about CMA: http://freesurfer.net/fswiki/CMA

Link to website archive: https://web.archive.org/web/20180413052010/http://www. cma.mgh.harvard.edul

Wakana, S. et al. Reproducibility of quantitative tractography methods applied to cerebral white matter. Neurolmage 36, 630-644 (2007).

Hua, K. et al. Tract probability maps in stereotaxic spaces: Analyses of white matter anatomy and tract-specific quantification. Neurolmage 39, 336-347 (2008).

MRI atlas of human white matter. (Elsevier, Acad. Press, 2011).

(1) Amunts, K., Mohlberg, H., Bludau, S. \& Zilles, K. Julich-Brain: A 3D probabilistic atlas of the human brain's cytoarchitecture. 6 (2020).

(2) Eickhoff, S. B. et al. A new SPM toolbox for combining probabilistic cytoarchitectonic maps and functional imaging data. Neurolmage 25, 1325-1335 (2005)

https://www.fz-juelich.de/inm/inm-1/DE/Forschung/_docs/SPMAnatomyToolbox/ SPMAnatomyToolbox_node.html

Glasser, M. F. et al. A multi-modal parcellation of human cerebral cortex. Nature 536, 171-178 (2016).

Coalson, T. S., Van Essen, D. C. \& Glasser, M. F. The impact of traditional neuroimaging methods on the spatial localization of cortical areas. Proc Natl Acad Sci USA 115, E6356-E6365 (2018).

https://balsa.wustl.edu/

Zalesky, A. et al. Whole-brain anatomical networks: does the choice of nodes matter? Neuroimage 50, 970-83 (2010).

(1) Goni, J. et al. Resting-brain functional connectivity predicted by analytic measures of network communication. Proceedings of the National Academy of Sciences 111, 833-838 (2014).

(2) Mišić, B. et al. Cooperative and Competitive Spreading Dynamics on the Human Connectome. Neuron 86, 1518-29 (2015). 


\section{Atlas}

Source Note

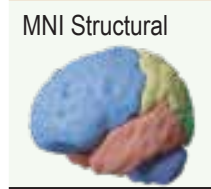

41

Schaefer

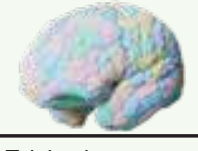

Talairach

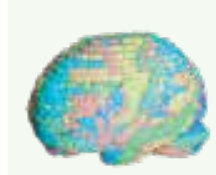

45

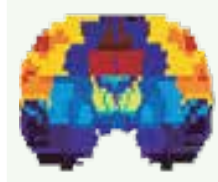

42

43

44

46
47

Original publication about functional parcellations.

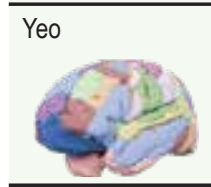

50

Website from FreeSurfer.

51

Thalamus - based on ex vivo analysis.

52

Hippocampus - based on ex vivo analysis.

Structural atlas of Cerebellum. Included with FSL.

54 Functional atlas of Cerebellum.

\section{Reference(s)}

(1) Website: http://www.talairach.org/about.html

(2) http://www.talairach.org/about.html

(3) Mazziotta, J. et al. A probabilistic atlas and reference system for the human brain: International Consortium for Brain Mapping (ICBM). Phil. Trans. R. Soc. Lond. B 356, 1293-1322 (2001).

Schaefer, A. et al. Local-Global Parcellation of the Human Cerebral Cortex from Intrinsic Functional Connectivity MRI. Cerebral Cortex 28, 3095-3114 (2018).

https://github.com/ThomasYeoLab/CBIG/tree/master/stable_projects/brain_parcellation/Schaefer2018_LocalGlobal

Website: http://www.talairach.org/

(1) Lancaster, J. L., Evans, A. C. \& Toga, A. W. Automated Labeling of the Human Brain: A Preliminary Report on the Development and Evaluation of a Forward-Transform Method. 238-242 (1997).

(2) Lancaster, J. L. et al. Automated Talairach Atlas Labels For Functional Brain Mapping. 120-131 (2000).

(1) Talairach, J., David, M., Tournoux, P., Corredor, H. \& Kvasina, T. Atlas d'Anatomie Stéréotaxique. Repérage Radiologique Indirect des Noyaux Gris Centraux des Régions Mésencephalosousoptique et Hypothalamique de l'Homme. (1957).

(2) Talairach, J. \& Szikla, G. Atlas of Stereotaxic Anatomy of the Telencephalon. (Masson, 1967)

(3) Talairach, J. \& Tournoux, P. Co-planar stereotaxic atlas of the human brain: 3-dimensional proportional system: an approach to cerebral imaging. (Georg Thieme, 1988).

Harary, M. \& Cosgrove, G. R. Jean Talairach: a cerebral cartographer. Neurosurgical Focus 47, E12 (2019).

Lancaster, J. L. et al. Bias between MNI and Talairach coordinates analyzed using the ICBM-152 brain template. Hum. Brain Mapp. 28, 1194-1205 (2007).

Thomas Yeo, B. T. et al. The organization of the human cerebral cortex estimated by intrinsic functional connectivity. Journal of Neurophysiology 106, 1125-1165 (2011)

https://surfer.nmr.mgh.harvard.edu/fswiki/CorticalParcellation_Yeo2011

Iglesias, J. E. et al. A probabilistic atlas of the human thalamic nuclei combining ex vivo MRI and histology. Neurolmage 183, 314-326 (2018).

Iglesias, J. E. et al. A computational atlas of the hippocampal formation using ex vivo, ultra-high resolution MRI: Application to adaptive segmentation of in vivo MRI. Neurolmage 115, 117-137 (2015).

Diedrichsen, J., Balsters, J. H., Flavell, J., Cussans, E. \& Ramnani, N. A probabilistic MR atlas of the human cerebellum. Neurolmage 46, 39-46 (2009).

(1) Xue, A. et al. The Detailed Organization of the Human Cerebellum Estimated by Intrinsic Functional Connectivity Within the Individual. 69.

(2) Buckner, R. L., Krienen, F. M., Castellanos, A., Diaz, J. C. \& Yeo, B. T. T. The organization of the human cerebellum estimated by intrinsic functional connectivity. Journal of Neurophysiology 106, 2322-2345 (2011).

(2) GitHub: https://github.com/ThomasYeoLab/CBIG/tree/master/stable_projects/ brain_parcellation/Xue2021_IndCerebellum

Alexander, B. et al. A new neonatal cortical and subcortical brain atlas: the Melbourne Children's Regional Infant Brain (M-CRIB) atlas. Neurolmage 147, 841-851 (2017). 